\title{
An introduction to mathematical models of coagulation-fragmentation processes: a discrete deterministic mean-field approach
}

\author{
Jonathan AD Wattis \\ Theoretical Mechanics, School of Mathematical Sciences, University of \\ Nottingham, University Park, Nottingham, NG7 2RD, UK.
}

24 July 2008

\begin{abstract}
We summarise the properties and the fundamental mathematical results associated with basic models which describe coagulation and fragmentation processes in a deterministic manner and in which cluster size is a discrete quantity (an integer multiple of some basic unit size). In particular, we discuss Smoluchowski's equation for aggregation, the Becker-Doring model of simultaneous aggregation and fragmentation, and more general models involving coagulation and fragmentation.
\end{abstract}

Key words: coagulation, aggregation, fragmentation, mathematical modelling PACS: 64.60.-i, 05.70.Fh, 05.70.Ln, 01.30.Rr, 02.30.Hq, 02.30.Jr.

\section{Introduction}

This paper gives an introductory overview of some mathematical models of phenomena which include coagulation and fragmentation. These phenomena, also referred to as aggregation, coalescence, or nucleation arise in many applications: for example, the formation of aerosols [1,2], colloidal aggregates $[3,4]$, polymers [5] and even celestial bodies on astronomical scales [6,7]. There are many approaches to modelling these phenomena, and here we shall focus on just one, that of discrete, deterministic (mean-field) models which can be traced back to the work of Smoluchowski [8] and Becker \& Döring [9]. Here, 'discrete' refers to the possible values taken by the cluster size. We assume

Email address: Jonathan.Wattis@nottingham.ac.uk (Jonathan AD Wattis).

Preprint submitted to Elsevier Science 24 July 2008 
that the system is composed of a large number of identical 'atoms', which are fundamental and indivisible units. We assume that atoms can bind to other atoms, hence forming clusters. The mass of each cluster is thus an integer multiple of some fundamental unit (the mass of the atom). We assume time to be a continuous variable. Other approaches treat both time and a cluster's mass as continuous parameters. Typically, we model some process by introducing variables $\left\{c_{r}(t)\right\}_{r=1}^{\infty}$ which represent the concentrations of clusters of size $r$ at time $t$, the concentrations being averaged over some spatial domain. We assume that clusters interact with each other according to the law of mass action, which introduces a constant of proportionality known as a rate constant. In general, this rate constant will depend on the sizes of both the clusters involved in the interaction. An alternative approach is to model coagulation and fragmentation as stochastic processes [10]. Space prevents us from giving a detailed derivation of such stochastic models.

The importance of deterministic mean field equations lies in their wide range of applicability. There is a considerable literature on the existence and uniqueness of solutions to discrete and continuous formulations of coagulation-fragmentation models, see for example [11-17]. A common feature of many models is self-similar behaviour. Both mathematicians and physicists are interested in techniques for finding self-similar solutions and rigorously determining the range of initial data for which they form the large-time attractors (see Sections 2.3 and 2.6 for details). We highlight the types of rate coefficients used in applications and discuss the large-time behaviour of solutions of the resulting equations, such as convergence to an equilibrium, or to a steady-state, or to some form of a self-similar solution. The distinction between the terms steadystate and equilibrium is outlined in Section 3.1.

In Section 2 we review Smoluchowski's coagulation equation derived in 1917 [8] and some of the more significant results obtained for it since. In Section 3 we discuss the Becker-Döring equations, derived in 1935 [9]. These equations include both coagulation and fragmentation. Before a brief final Conclusions section, we discuss, in Section 4, more general models which include combinations of coagulation and fragmentation mechanisms. While more complicated than the Smoluchowski or the Becker-Döring systems, these provide more accurate descriptions of phenomena observed in practical applications. 


\section{Smoluchowski's coagulation equation}

\subsection{Formulation}

Smoluchowski's coagulation equation describes the kinetics of the process of binary aggregation. Consider a well-stirred vessel in which clusters of a variety of sizes move about and occasionally collide and coalesce with each other. We wish to construct a set of differential equations which model how the cluster-size distribution changes over time. Denoting a cluster composed of $r$ fundamental units by $C_{r}$, this process can be represented by

$$
C_{r}+C_{s} \rightarrow C_{r+s}
$$

If this reaction occurs with a rate constant $a_{r, s}$ and $c_{r}(t)$ denotes the concentration at time $t$ of $C_{r}$, the law of mass action yields the equations

$$
\frac{\mathrm{d} c_{r}}{\mathrm{~d} t}=\frac{1}{2} \sum_{s=1}^{r-1} a_{s, r-s} c_{s} c_{r-s}-\sum_{s=1}^{\infty} a_{r, s} c_{r} c_{s}
$$

The aggregation rate coefficients, $a_{r, s}$, are also referred to collectively as the 'aggregation kernel' or simply 'kernel'. The factor of $1 / 2$ is included to avoid double-counting, since the first sum includes every possible way of constructing the cluster $C_{r}$ twice. This model assumes that there are no spatial correlations in the vessel and that two clusters of any size can coalesce. The equation for the monomer concentration $(r=1)$ is obtained by ignoring the first sum in $(2.2)$, that is

$$
\frac{\mathrm{d} c_{1}}{\mathrm{~d} t}=-\sum_{s=1}^{\infty} a_{1, s} c_{1} c_{s}
$$

Since (2.1) is irreversible, the system has no equilibrium configuration, a property shared by the mathematical model (2.2)-(2.3).

The Smoluchowski coagulation equations can be derived in an alternative manner. Starting from a Markov process, one can define a master equation, from which the behaviour of fluctuations can be analysed as well as the expected behaviour of the coagulation process. For details of this approach see the review of Aldous [10], the recent work of Norris [18] and van Dongen \& Ernst $[19,20]$.

We aim to determine whether the evolution equations (2.2)-(2.3) conserve the total mass in the system, and describe the existence and form of self-similar so- 
lutions (and their basins of attraction). In the next two subsections we explain these concepts, before analysing three special aggregation kernels in detail. As well as yielding exact solutions, these solutions provide instructive illustrations of these concepts. Finally we comment on the role of mass conservation and similarity solutions in systems with more general aggregation kernels.

\subsection{Mass conservation}

Since each cluster $C_{r}$ is composed of $r$ units, and due to the conservation of mass in the reaction mechanism (2.1), we might expect the total mass of the system, defined by

$$
M_{1}(t)=\sum_{r=1}^{\infty} r c_{r}(t),
$$

to be conserved by (2.2)-(2.3). However, this is not always true; in Section 2.4.3 we will construct an exact solution to (2.2)-(2.3) for which the total mass (2.4) decreases. Many authors prefer 'density' instead of mass, and consequently use the symbol $\varrho$ in place of $M_{1}$.

To analyse the effect of loss of mass in more detail, we follow Davies et al. [21] and introduce a mass flux, $J_{r}$, from clusters of size at most $r$ to clusters of size larger than $r$ by

$$
J_{r}=\sum_{j=1}^{r} \sum_{k=r+1-j}^{\infty} j a_{k, j} c_{j} c_{k},
$$

together with $J_{0}=0$. This definition implies that

$$
\frac{\mathrm{d}\left(r c_{r}(t)\right)}{\mathrm{d} t}=J_{r-1}-J_{r},
$$

an equation which is similar to the Becker-Döring models considered in the next section (see equation (3.3), for example). Summing these equations over $r$ leads to

$$
\frac{\mathrm{d} M_{1}}{\mathrm{~d} t}=-J_{\infty}:=-\lim _{r \rightarrow \infty} J_{r}
$$

In systems where the cluster distribution function $c_{r}$ decays rapidly enough with increasing $r$ (for example, exponentially), $J_{\infty}$ is zero and $M_{1}(t)$ is conserved. However, it is possible that the coagulation process alters the decay of 
the size distribution $c_{r}(t)$ so that after some time it becomes algebraic in $r$, and then for certain exponents $\left(\gamma\right.$, where $c_{r} \sim C_{0} r^{-\gamma}$ as $\left.r \rightarrow \infty\right)$ the limit (2.7) may be nonzero. If $J_{\infty}$ is non-zero, there is a flux of mass out of the system, a flux to a particle of infinite size, which is known as the 'superparticle', or more commonly, the 'gel'. The creation of such a particle makes (2.2) a good model for certain phase transitions. In equation (2.35), a specific kernel is introduced and used to illustrate the calculation of $J_{\infty}$.

In systems where $M_{1}$ is not conserved, it is useful to define the gelation time, or gel-point, $t_{g} \in[0,+\infty]$, which is the earliest time where conservation of $M_{1}$ fails:

$$
t_{g}:=\inf \left\{t>0: M_{1}(t)<M_{1}(0)\right\}
$$

\subsection{Similarity solutions}

In any particular application, determination of the form of the rates $a_{r, s}$ is not a trivial task. In general these rate constants include information on the rate at which clusters move around the system under consideration, as well as the likelihood of clusters coagulating if they meet. Note that in the Smoluchowski model the only characteristic of a cluster we retain is its mass. Thus any variations in shape (morphology) among clusters of the same size have to be averaged over when calculating the coagulation rates $a_{r, s}$.

Many different aggregation kernels $a_{r, s}$ have been used to model coagulation phenomena. For example, the case of size-independent coagulation rates, where $a_{r, s}=a$, can be used to model approximate Brownian coagulation [22], the kernel $a_{r, s}=a(r+s)$ arises as a limit case of gravitational coagulation [22], whilst $a_{r, s}=$ ars occurs in branched chain polymerisation [5,23]. These three specific examples will be studied in greater detail in Sections 2.4.1, 2.4.2 and 2.4.3. In [24], da Costa has summarised a wide range of rate coefficients. Derivations for some of the simpler cases are given by Collet [25].

As well as the natural symmetry condition $a_{r, s}=a_{s, r}$, in many applications the aggregation kernel has a self-similar form, obeying the homogeneity condition $a_{k r, k s}=k^{\lambda} a_{r, s}$; hence $a_{r, s}=r^{\lambda} a_{1, s / r}=r^{\lambda} \alpha(r / s)$ for some function $\alpha(\cdot)$. Given this scaling condition, it is natural to wonder whether there exist similarity solutions of (2.2)-(2.3). In general, we seek similarity solutions of the form

$$
c_{r}(t)=k(t) g(\eta) \quad \text { with } \quad \eta=\eta(r, t)=\frac{r}{h(t)}
$$

Two special subsets of such solutions most commonly arise. The first, known as dynamical scaling solutions, have $h(t)$ nonconstant, say $h(t)=s(t)$ where 
$s(t)$ is a typical cluster size, with $s(t) \rightarrow \infty$ as $t \rightarrow t_{1}$ ( $t_{1}$ being some constant, possibly infinite). Then $k(t)=s(t)^{-\tau}$, and we have the form

$$
c_{r}(t) \sim \frac{1}{s(t)^{\tau}} g\left(\frac{r}{s(t)}\right) .
$$

Due to $\eta=r / s(t)$ being a continuous variable, the sum in the definition of $M_{1}(2.4)$ is replaced by an integral over $g(\eta)$. Equation (2.10) implies that $M_{1}(t)=s(t)^{2-\tau} \int_{0}^{\infty} \eta g(\eta) \mathrm{d} \eta$, and so mass conservation imposes the restriction $\tau=2$. However, we have already noted above that the total mass is not necessarily conserved, and so solutions with $\tau \neq 2$ could arise. The second special form of similarity solution is the separable solution, in which $h(t)=1$. Solutions of this type therefore take the form $c_{r}(t)=f_{r} / t, f_{r} \in \mathbb{R}$ for all $r \geq 1$. We thus find that all terms in $(2.2)-(2.3)$ have the factor $(1 / t)^{2}$, and the problem is then to determine $f_{r}$; with there being no guarantee that a solution for $f_{r}$ actually exists. If such a solution does exist, the mass is given by $M_{1}(t)=\left(\sum_{r=1}^{\infty} r f_{r}\right) / t$.

Having raised these issues, the natural questions are the following. For which aggregation kernels or initial data is mass conservation assured? Under what conditions does it fail? For which kernels/initial data do solutions approach a similarity solution? We will return to these questions in Sections 2.5 and 2.6, after we have studied some specific examples in detail.

\subsection{Systems with explicit solutions}

The results below are derived using the generating function approach of Davies et al. [21], though the results themselves were originally found by other authors, often using different methods. We define

$$
C(z, t)=\sum_{r=1}^{\infty} c_{r}(t) \mathrm{e}^{-r z}
$$

so that $M_{0}(t)=C(0, t)=\sum_{r=1}^{\infty} c_{r}(t)$ is the total number of clusters in the system, $M_{1}(t)=-C_{z}(0, t)=\sum_{r=1}^{\infty} r c_{r}(t)$ is the total mass of the system and the $k^{\text {th }}$ moment of the cluster size distribution function is

$$
M_{k}(t)=\left.(-1)^{k} \frac{\partial^{k}}{\partial z^{k}} C(z, t)\right|_{z=0 .}
$$

The function $C(z, t)$ transforms the system of coupled ordinary differential equations (2.2) into a single partial differential equation. 
For simplicity of the calculations in this section, we will assume monodisperse initial conditions, that is, the system has all its (unit) mass in monomeric form

$$
c_{r}(0)=0 \quad \text { for all } r>1, \quad c_{1}(0)=1
$$

This implies that the equation for the generating function should be solved subject to the initial data $C(z, 0)=\mathrm{e}^{-z}$. The evolution of other initial data may be more complex, and results for such cases will be discussed in more detail after equation (2.18) and in Section 2.6.

\subsubsection{Explicit solution I: the constant kernel, $a_{r, s}=1$}

Using the generating function (2.11), we have in this case

$$
\frac{\partial C}{\partial t}=\frac{1}{2} C(z, t)^{2}-C(0, t) C(z, t)
$$

and thus we need to find $C(0, t)=M_{0}(t)$ before we can proceed. Putting $z=0$ into (2.14), we find a first-order ordinary differential equation for the total number of clusters in the system, $M_{0}(t)$, namely $\dot{M}_{0}=-\frac{1}{2} M_{0}^{2}$. From (2.13), the initial data is $M_{0}(0)=1$, implying that

$$
M_{0}=\frac{2}{t+2}
$$

The Riccati equation (2.14) is then solved by the transformation $Y(z, t)=$ $1 / C(z, t)$ which gives a linear problem for $Y(z, t)$. Imposing the initial data $Y(z, 0)=1 / C(z, 0)=\mathrm{e}^{z}$, we obtain the solution

$$
C(z, t)=\frac{4 \mathrm{e}^{-z}}{(t+2)^{2}}\left(1-\frac{t \mathrm{e}^{-z}}{t+2}\right)^{-1} .
$$

An expansion of the final term as a power series yields the solution

$$
c_{r}(t)=\frac{4}{(t+2)^{2}}\left(\frac{t}{(t+2)}\right)^{r-1}, \quad t \geq 0 .
$$

Using (2.12), we see that $M_{1}(t)=1, M_{2}(t)=1+t$, and $M_{0}$ is given by (2.15). Thus, whether one defines the typical cluster size by $M_{1} / M_{0}=1+\frac{1}{2} t$ or by $M_{2} / M_{1}=1+t$, we find that the typical cluster size grows linearly with time. For $t \gg 1$ and $r=O(t)$ the solution (2.17) has the form

$$
c_{r}(t)=\frac{4}{t^{2}} \mathrm{e}^{-2 r / t} \quad \text { as } \quad t \rightarrow \infty \text { with } \quad r=O(t) .
$$


Note that this is of the form (2.10) with $\tau=2$ and $s(t)=t$.

A similarity solution of the form $c_{r}(t)=(1 / t)^{2} g(r / t)$ has been postulated for some time, and proven to exist for certain classes of initial data. Kreer \& Penrose [26] and da Costa [27] prove the result for initial data which are at most exponentially decaying with cluster size; more recently, Menon \& Pego [28-30] have proved similar results involving algebraically decaying initial data. The existence of a self-similar distribution means that the system loses knowledge of the details of its initial data. Provided the initial mass is known, and that the initial conditions are compactly supported, the solution at large times can be given in a particularly simple form.

\subsubsection{Explicit solution 2: the additive kernel, $a_{r, s}=(r+s) / 2$}

The partial differential equation for the generating function $C(z, t),(2.11)$, is now

$$
\frac{\partial C}{\partial t}=-\frac{1}{2} C \frac{\partial C}{\partial z}+\frac{1}{2} \frac{\partial C}{\partial z} M_{0}(t)-\frac{1}{2} M_{1}(t) C
$$

By setting $z=0$ in (2.19), we have $\dot{M}_{0}=-\frac{1}{2} M_{0} M_{1}$. Differentiating (2.19) with respect to $z$, and putting $u=-C_{z}$ we obtain

$$
u_{t}=\frac{1}{2} u\left(u-M_{1}\right)+\frac{1}{2} u_{z}\left(M_{0}-C\right) .
$$

Setting $z=0$ gives $\dot{M}_{1}=0$ (provided neither $u$ nor $u_{z}$ diverge at $z=0$ ) so we have $M_{1}(t)=1$ for all time. The equation for $M_{0}$ can then be solved to show that $M_{0}(t)=\mathrm{e}^{-t / 2}$. The equation for the second moment, $M_{2}$, is $\dot{M}_{2}=M_{1} M_{2}$; thus $M_{2}=\mathrm{e}^{t}$ and both $u$ and $u_{z}$ are bounded at $z=0$ (since $M_{1}=u(0, t)$ and $\left.M_{2}=-u_{z}(0, t)\right)$.

Using these results, equation (2.19) can be rewritten as

$$
\frac{\partial C}{\partial t}=\frac{1}{2}\left(\mathrm{e}^{-t / 2}-C\right) \frac{\partial C}{\partial z}-\frac{1}{2} C
$$

which can be solved in implicit form using the method of characteristics to give

$$
\mathrm{e}^{-z}=C(z, t) \exp \left(1+\frac{1}{2} t-\mathrm{e}^{-t / 2}\right) \exp \left(-\left(\mathrm{e}^{t / 2}-1\right) C(z, t)\right)
$$


Using Lagrange's expansion ([31] p.14, eq. 3.6.6) the concentrations can then be shown to be ${ }^{1}$

$$
c_{r}(t)=\frac{1}{r !} r^{r-1} \mathrm{e}^{-t / 2}\left(1-\mathrm{e}^{-t / 2}\right)^{r-1} \exp \left(-r\left(1-\mathrm{e}^{-t / 2}\right)\right)
$$

For a typical cluster size we take $M_{2} / M_{1}=\mathrm{e}^{t}$. To investigate the existence of a similarity solution valid at large times and for large aggregation numbers, we put $r=\eta \mathrm{e}^{t}$, and expand (2.23) for large $t$, assuming $\eta$ to be $O(1)$. Making use of Stirling's formula ([31], p.256-7, equations $6.1 .37 \& 6.1 .15$ ), we obtain the similarity solution

$$
c_{r}(t) \sim \mathrm{e}^{-2 t} g\left(\frac{r}{\mathrm{e}^{t}}\right), \quad \text { with } \quad g(\eta)=\frac{\mathrm{e}^{-\eta / 2}}{\left(2 \pi \eta^{3}\right)^{1 / 2}} .
$$

Note that this has the form (2.10) with $\tau=2$ and $s(t)=\mathrm{e}^{t}$.

\subsubsection{Explicit solution 3: the product kernel, $a_{r, s}=r s$}

The final exactly solvable kernel which we consider here is the product kernel, which yields a different type of behaviour in the large time limit. In this case, from (2.11) we obtain the equation

$$
\frac{\partial C}{\partial t}=\frac{1}{2}\left(\frac{\partial C}{\partial z}\right)^{2}+M_{1}(t) \frac{\partial C}{\partial z}
$$

for the generating function. The substitution $u(z, t)=-C_{z}(z, t)$ simplifies this to an inviscid Burgers equation

$$
\frac{\partial u}{\partial t}=\left(M_{1}(t)-u\right) \frac{\partial u}{\partial z}
$$

Menon and Pego [28,29], and Bertoin [32] have also demonstrated underlying links between the Smoluchowski coagulation equation and the inviscid Burgers equation. Solving (2.26) by the method of characteristics, subject to $u(z, 0)=$

$\overline{1 \text { This }}$ derivation uses $y=e^{-z}, x=C, x_{0}=0=y_{0}, f(x)=A x e^{-b x}$, where $A=e^{1+t / 2-e^{-t / 2}}$ and $b=e^{t / 2}-1$. We then have

$$
C=x=\sum_{k=1}^{\infty} \frac{y^{k}}{k !}\left[\frac{\mathrm{d}^{k-1}}{\mathrm{~d} x^{k-1}}\left(\frac{x}{A x \mathrm{e}^{-b x}}\right)^{k}\right]_{x=0}=\sum_{k=1}^{\infty} \frac{e^{-k z}}{k !} A^{-k}(b k)^{k-1} .
$$


$\mathrm{e}^{-z}$, yields

$$
z=t u-\log u-\int_{0}^{t} M_{1}\left(t^{\prime}\right) \mathrm{d} t^{\prime},
$$

so that

$$
\frac{\partial u}{\partial z}=-\frac{u}{1-t u}
$$

It remains to determine $M_{1}(t)$. Since $u(0, t)=M_{1}(t)$, it follows from $(2.26)$ that there are two possibilities:

(i) the 'pre-gelation' case, valid at times earlier than a critical value, which, for the time being, we shall define as $t_{c}$; (later, we will show that this time, $t_{c}$, is actually the gelation time, $t_{g}$, defined by (2.8)). For $0 \leq t<t_{c}$ we have $\dot{M}_{1}=0$ and $u(0, t)=M_{1}=1$. The line $z=0$ is thus a characteristic of (2.26), up until the critical time $t=t_{c}$. Equation (2.27) implies

$$
z=t u-\log u-t
$$

and (2.28) evaluated at $z=0$ implies that

$$
M_{2}(t)=\frac{1}{1-t} \quad \text { for } \quad t<1
$$

However, as $t \rightarrow 1^{-}, M_{2}(t)$ blows up; we thus define $t_{c}=1$. For $t>t_{c}=1$, $u(z, t)$ as given by (2.29) is multi-valued in $z>0$, implying the presence of a shock in the solution to $(2.26)$.

(ii) Since $M_{2}(t)=-u_{z}(0, t) \rightarrow \infty$ as $t \rightarrow t_{c}^{-}, u(z, t)$ develops a shock at $t=t_{c}$ and $z=0$, where $\frac{\partial u}{\partial z}(0, t)$ is unbounded. For $t>t_{c}$, we determine $M_{1}(t)=u(0, t)$, by putting $z=0$ into $(2.28)$, requiring the singularity in $u_{z}$ to remain at $z=0$, hence $u(0, t)=1 / t$ for $t>t_{c}$, and so $M_{1}=u(0, t)$ is genuinely a time-dependent quantity in $t>t_{c}$. Since $t=t_{c}$ is the first point at which mass is lost from the system, applying the definition (2.8) we deduce that $t_{g}=t_{c}$.

Equation (2.27) then gives

$$
z=t u-\log u-1-\log t
$$


Combining (2.29) and (2.31) we obtain

$$
\exp (-z)= \begin{cases}u \exp ((1-u) t) & t \leq 1 \\ u t \exp (1-u t) & t \geq 1\end{cases}
$$

from which $u(z, t)$ can be extracted using Lagrange's Expansion [31], ultimately giving

$$
c_{r}(t)= \begin{cases}\frac{r^{r-3} t^{r-1} \mathrm{e}^{-r t}}{(r-1) !} & t \leq 1 \\ \frac{r^{r-3} \mathrm{e}^{-r}}{(r-1) ! t} & t \geq 1 .\end{cases}
$$

The transition to a mass-losing solution at $t=1$ is referred to as gelation . This change in the solution at a critical time was first identified by Ziff \& Stell [33] and Leyvraz \& Tschudi [34,35].

The post-gelation behaviour $\left(t>t_{g}:=1\right)$ is exactly of the separable form $c_{r}(t)=f_{r} / t$. From this we conclude that the total mass of the system also varies with $t$, scaling according to $M_{1}(t)=1 / t$ for $t>t_{g}:=1$. Prior to gelation, $M_{1}(t)=1$, so $M_{1}(t)$ is continuous across the gel-point $t=t_{g}=t_{c}=1$ (see Figure 1).

By applying Stirling's formula to (2.33), the large $r$ behaviour can be written in the form

$$
c_{r}(t) \sim \begin{cases}\frac{\mathrm{e}^{-r(t-1-\log (t))}}{r^{5 / 2} t \sqrt{2 \pi}} & t<1 \\ \frac{1}{r^{5 / 2} t \sqrt{2 \pi}} & t \geq 1 .\end{cases}
$$

After gelation and for large $r$, we have $f_{r} \sim 1 / \sqrt{2 \pi} r^{5 / 2}$. Equation (2.34) illustrates that for $t<1$ the cluster size distribution function decays exponentially, with rate $(t-1-\log (1-t))$. However, as $t \rightarrow 1^{-}$, this rate vanishes, and at $t=1$, the decay is only algebraic, with power $-5 / 2$. After gelation, the size distribution retains this algebraic decay, with the same exponent. These cluster-distribution functions are plotted in Figure 1. Just prior to gelation, both algebraic and exponential behaviour can be seen in the tail of the distribution: when $t=1-\varepsilon$ with $0<\varepsilon \ll 1, \log c_{r}(t) \sim-\frac{1}{2} \varepsilon^{2} r-\frac{5}{2} \log r$, so that if $r$ is of intermediate-large size $(r \gg 1)$ with $\log r \gg \varepsilon^{2} r$ (which can be rewritten as $1 \ll r \ll \varepsilon^{-2}$ ), algebraic decay is observed. However, if $r$ is extremely large, so that $\varepsilon^{2} r \gg \log r$, then exponential decay is seen. 


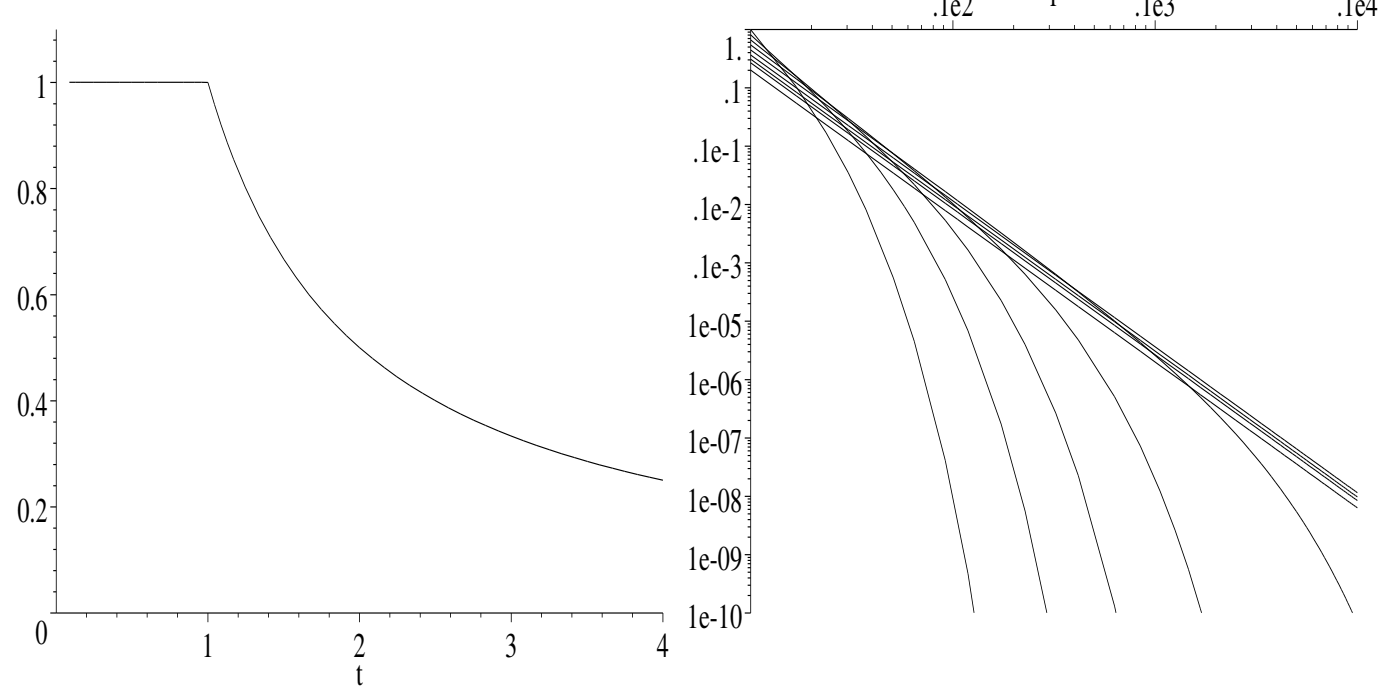

Fig. 1. Left: graph of total mass $M_{1}(t)$ against time, showing delayed gelation occurring at $t=1$. Right: $\log$-log plot of cluster distribution function $c_{r}(t)(2.34)$ against size $(r)$ for $1<r<10^{4}$ at times $t=0.1,0.3,0.5,0.7,0.9,1.1,1.3,1.5,2$ showing algebraic decay (straight lines) for times $t \geq 1$ and exponential decay at larger $r$ for earlier times $(t<1)$.

\subsection{Gelation and mass conservation in more general kernels}

So far we have considered only three very special coagulation kernels and have focused on monodisperse initial data. As a step on the way to analysing a broader range of kernels and more general initial conditions, we now illustrate how approximate methods can be used to show that behaviour similar to that observed above also occurs in systems with more general aggregation kernels. In equations (2.18), (2.24) and (2.34) we have shown that the exact solutions converge to self-similar solutions in the limit of large times and large cluster sizes.

If we are only interested in the asymptotics of the solution at large-times and large-cluster sizes then we only need to know the form of the kernel $a_{r, s}$ at large sizes (as $r$ and/or $s \rightarrow \infty$ ). Thus it is sufficient to consider kernels of the form

$$
a_{r, s}=a\left(r^{\mu} s^{\nu}+r^{\nu} s^{\mu}\right)
$$

for $r, s \gg 1$, with $a \in \mathbb{R}_{+}$. If we assume that the aggregation rate kernel is given by (2.35) and that cluster size distribution decays algebraically, via $c_{r} \sim r^{-\gamma}$ as $r \rightarrow \infty$, then we find that requiring the inner sum (over $k$ ) of (2.5) to converge implies that $\gamma>1+\mu$ and $\gamma>1+\nu$. Evaluating the outer sum yields $J_{r} \sim r^{3+\mu+\nu-2 \gamma}$ so for gelation to occur, we require $\gamma=(\mu+\nu+3) / 2$. This calculation is confirmed by (2.34) for the exactly solvable case $\mu=\nu=1$. 


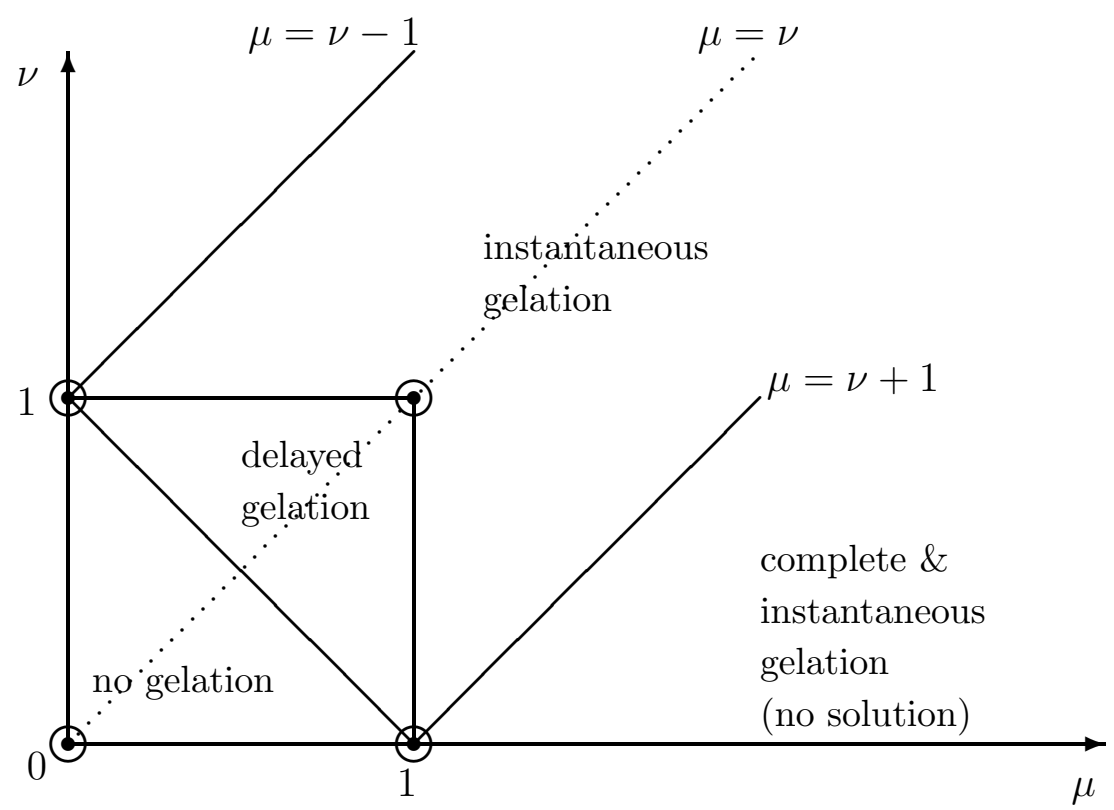

Fig. 2. The four types of gelation behaviour for $a_{r, s}=a\left(r^{\mu} s^{\nu}+r^{\nu} s^{\mu}\right)$. Note that the exactly solvable cases located at $(\mu, \nu)=(0,0),(1,0),(0,1)$ and $(1,1)$ (marked by $\odot)$ all lie at the boundaries between two or more regions of different generic behaviour.

If $\mu+\nu<1$ then $\gamma<2$ and the mass of any distribution with $c_{r} \sim r^{-\gamma}$ as $r \rightarrow \infty$ is divergent. Hence no initial distribution of finite mass can adopt the shape required for gelation to occur, and such systems cannot undergo gelation. However, if $\mu+\nu>1$ then $\gamma>2$ and distributions of the form $c_{r} \sim r^{-\gamma}$ have finite mass; consequently it is possible for gelation to occur when $\mu+\nu>1$.

For the set of rates given by (2.35), one of four types of behaviour is observed $[21,36]$. For an illustration of the regions of $(\mu, \nu)$-parameter space where each occurs, see Figure 2. We define $\lambda=\mu+\nu$. The four behaviours are

- no gelation, when $0<\mu<1,0<\nu<1$ and $\lambda<1$; this is similar to the behaviour observed in the constant kernel and the additive kernel (Cases I and II),

- delayed gelation, as observed in the product kernel (Case III, Section 2.4.3), when the total mass $M_{1}(t)$ is conserved for a finite time $\left(0 \leq t \leq t_{g}\right)$, after which $M_{1}(t)$ decays in time; this occurs when $0<\mu<1,0<\nu<1$ and $\lambda>1$, (for certain initial data, $t_{g}$ may be zero),

- instantaneous gelation for any initial data, by which we mean that the total mass $M_{1}(t)$ decreases from $t=0$. This occurs when $-1<\mu-\nu<1$ and $\max \{\mu, \nu\}>1$,

- non-existence of solutions due to complete and instantaneous gelation; this occurs for $|\mu-\nu|>1$. Here, numerical simulations of truncated systems with monomeric initial data show a significant proportion of the mass being 
transported from the monomers to the largest cluster sizes over a very few timesteps (no matter how small the timestep is chosen).

From numerical investigation of the coagulation equations, Lee [37] notes that in the example of (2.35) instantaneous gelation occurs when $\nu>1$ or $\mu>1$. However, in [37] no distinction is made between the cases of instantaneous gelation for $|\mu-\nu|<1$ and 'complete and instantaneous gelation' for $|\mu-\nu|>$ 1. Van Dongen [36] notes that this latter case corresponds to non-existence of solutions to the full (non-truncated) system of equations. This is due to the instantaneous transfer of mass from the sol to the gel. Carr \& da Costa [38] prove that instantaneous gelation occurs for any non-zero initial condition if the rate coefficients have the form $r^{\alpha}+s^{\alpha}<a_{r, s}<(r s)^{\beta}$ with $\beta>\alpha>1$.

The problems in proving the existence of a physically meaningful solution of an infinite set of coupled nonlinear ordinary differential equations are obvious. Existence and uniqueness results are slightly more straightforward for systems which cannot undergo gelation. Therefore many theorems are restricted to the case $a_{r, s}<a(r+s)$; see for example White [17], and Ball \& Carr [11]. In these cases it is possible to prove that the cluster distribution function $\left(c_{r}(t)\right)$ always decays exponentially at large sizes (as $r \rightarrow \infty)$. When kernels which permit gelation are considered, the problem becomes more difficult. McLeod $[14,15]$ first realised that for the product kernel $\left(a_{r, s}=\right.$ ars $)$ the first moment could not be conserved for all time.

Jeon [39] proved, via a probabilistic approach, the occurrence of gelation in more general Smoluchowski coagulation problems with kernels of the form $\varepsilon(r s)^{\alpha} \leq a_{r, s} \leq K r s$ with $\frac{1}{2}<\alpha \leq 1, \varepsilon>0, M_{1}<\infty$. Escobedo et al. [40] prove that gelation occurs in systems of the form (2.35) with $\lambda>1$, and $M_{1}(t) \leq C_{*} /(1+t)^{1 / \lambda}$, and that consequently the gelation time satisfies $t_{g}<\left(C_{*} / M_{1}(0)\right)^{\lambda}$. Leyvraz [41] and van Roessel \& Shirvani [42] give more detailed analyses of the form of the solution in the post-gelation phase of the reaction for more general aggregation kernels.

\subsection{Further results on similarity solutions}

Recently there has been a number of results proving convergence to similarity solutions for various kernels and various classes of initial data. Fournier \& Laurençot [43] consider the existence of similarity solutions for general homogeneous kernels with degree $\lambda<1$. As this condition prevents gelation, their similarity solutions are of the mass-conserving type (that is, $\tau=2$ in (2.10)). In particular they prove that for the continuous formulation of the Smoluchowski coagulation equations the kernels 


$$
\begin{aligned}
& a(r, s)=\left(r^{\alpha}+s^{\alpha}\right)\left(r^{-\beta}+s^{-\beta}\right), \quad 0 \leq \alpha<1, \quad \beta>0, \quad \lambda=\alpha-\beta<1, \\
& a(r, s)=\left(r^{\alpha}+s^{\alpha}\right)^{\beta}, \quad \alpha \geq 0, \quad \beta>0, \quad \lambda=\alpha \beta<1, \\
& a(r, s)=r^{\alpha} s^{\beta}+r^{\beta} s^{\alpha} \quad \alpha, \beta, \lambda=\alpha+\beta \in(0,1),
\end{aligned}
$$

there exists a similarity solution of the form (2.10) with $s(t)=t^{1 /(1-\lambda)}$ for the continuous formulation of Smoluchowski's coagulation equation. Their results show that the similarity function $g(\eta)$ decays rapidly as $\eta \rightarrow \infty$, and so is relevant for compact initial data. However, their results do not rule out the existence of other similarity solutions with weaker decay rates.

Menon \& Pego [28,29] have proved that for the continuous Smoluchowski coagulation equations with constant kernel, (the same kernel as analysed in Section 2.4.1), the system with initial data of finite total mass will converge to a similarity solution of the form (2.18). However, they also find similarity solutions of a different form, which have 'fat tails', that is, they decay slowly at large cluster sizes, so their total mass is divergent. They also find similarity solutions with divergent total mass for the product kernel (Section 2.4.3). These are related to another class of similarity solutions, which occur in the sum kernel (Section 2.4.2), and were discovered by Bertoin [32]. Due to a 'fat tail', these solutions have finite mass but a divergent second moment. Despite this, the system does not undergo gelation. For this kernel there are thus many similarity solutions which could be approached in the large-time limit. The solution approached depends on the decay rate of the initial data $c_{r}(0)$ as $r \rightarrow \infty$. We expect that similar results to these and to those of Fournier \& Laurençot discussed above will also hold for the discrete formulation of the Smoluchowski coagulation equations.

For more details on other results involving self-similarity and the asymptotic structure of solutions we refer the reader to the detailed study by Hendricks et al. [44] and the recent review by Leyvraz [45].

\subsection{Summary}

In this section we have introduced the Smoluchowski equations describing irreversible aggregation and, by solving explicitly a number of cases, noted that a variety of behaviours is observed at large times. The form of the distribution of large-cluster sizes at large times depends on the form of the aggregation kernel. In both the cases $a_{r, s}=1$ and $a_{r, s}=(r+s) / 2$, we have seen selfsimilar scaling behaviour in the large time limit for initial data of the form $c_{1}(0)=1, c_{r}(0)=0$ for all $r \geq 2$. This has the form $c_{r}(t)=\left(1 / s(t)^{\tau}\right) g(r / s(t))$. The function $s(t)$ is simply $t$ for the constant kernel and $s(t)=\mathrm{e}^{t}$ for the additive kernel. Since $\tau=2$ in both cases, these solutions preserve the mass

of the system, that is $M_{1}(t)$, given by $(2.4)$, is independent of time. At large 
time, $s(t) \rightarrow \infty$, indicating that the cluster-size distribution becomes slowly varying in $r$, and so the sum (2.4) can be replaced by the integral condition $M_{1}=\int_{0}^{\infty} \eta g(\eta) \mathrm{d} \eta=O(1)$. For both kernels, the shape of the distribution $g(\eta)$ decays exponentially in $\eta$, with $g(\eta)=\mathrm{e}^{-2 \eta}$ when $a_{r, s}=1$ and $g(\eta)=$ $\mathrm{e}^{-\eta / 2} / \eta^{3 / 2}$ for the additive kernel. In the case of $a_{r, s}=$ ars, we have noted that the phenomenon of gelation occurs, in which mass is lost from the 'finite' part of the system, that is the concentrations $\left\{c_{r}(t)\right\}_{r=1}^{\infty}$, and a particle of infinite size - the superparticle or gel - is formed at a definite time $\left(t=t_{g}\right)$ and then continues to grow. Although defined in terms of $M_{1}(t)$, the gelation time can often be determined by analysing the second moment, $M_{2}(t)$. Equation (2.26) evaluated at $z=0$ determines the evolution of $M_{1}(t)$ : at earlier times, $t<t_{g}$ we have $\dot{M}_{1}=0$ and so $M_{1}$ is conserved; after gelation, $\dot{M}_{1}$ is nonzero due to $u_{z}(0, t)=-M_{2}(t)$ being divergent. We have noted some of the features of Smoluchowski equations pertinent to more general rate kernels and have referred to papers which rigorously establish some of the more important phenomena, such as existence of solutions, mass-conservation, gelation, and self-similar behaviour.

\section{The Becker-Döring equations}

\subsection{Formulation}

The Becker-Döring equations model stepwise aggregation and fragmentation, that is, the loss or gain of a single particle at a time according to

$$
C_{r}+C_{1} \rightleftharpoons C_{r+1} \quad\left\{\begin{array}{l}
\text { forward rate }=a_{r} \\
\text { backward rate }=b_{r+1}
\end{array}\right.
$$

The inclusion of a fragmentation process, by allowing the coagulation process to be reversible introduces an additional complexity which we did not have to consider in (2.1). However, in compensation for this, the general aggregation reaction (2.1) is greatly simplified in that only cluster-monomer interactions are now allowed which removes the summations from (2.2)-(2.3). For example, the only reactions which involve $C_{4}$ are $C_{3}+C_{1} \rightleftharpoons C_{4}$ and $C_{4}+C_{1} \rightleftharpoons C_{5}$. Thus for the rate of change of the concentration of $C_{4}, c_{4}(t)$, we obtain the equation

$$
\frac{d c_{4}}{d t}=\underbrace{a_{3} c_{3}(t) c_{1}(t)-b_{4} c_{4}(t)}_{J_{3}} \underbrace{-a_{4} c_{4}(t) c_{1}(t)+b_{5} c_{5}(t)}_{-J_{4}} .
$$


In general, the concentrations $\left\{c_{r}(t)\right\}_{r=2}^{\infty}$ are governed by

$$
\frac{d c_{r}}{d t}=J_{r-1}(t)-J_{r}(t), \quad(r \geq 2)
$$

where the fluxes $J_{r}(t)$ are defined by

$$
J_{r}(t)=a_{r} c_{r}(t) c_{1}(t)-b_{r+1} c_{r+1}(t)
$$

This quantity is the net rate at which clusters of size $r+1$ are created from clusters of size $r$. If the fragmentation term $\left(b_{r+1} c_{r+1}\right)$ is larger than the aggregation term $\left(a_{r} c_{r} c_{1}\right)$ then $J_{r}$ will be negative.

We have not yet given an equation for the monomer concentration $c_{1}(t)$. There are various options. We could allow $c_{1}(t)$ to vary and specify an equation for it; for example, in Section 3.4, we consider a formulation in which $c_{1}(t)$ is allowed to vary and the total mass of the system, $M_{1}$, is fixed. Since $c_{1}(t)$ is then one of the unknowns, the problem becomes nonlinear and much harder to analyse. Alternatively, and more simply, we treat $c_{1}$ as a fixed parameter, $c_{1}(t) \equiv$ $c_{1}$. The assumption of a constant monomer concentration is valid in many circumstances, for example, systems into which monomers are continuously being added, or where there is some precursor species which breaks down so as to replenish the stock of monomers. For the remainder of this subsection and in Sections 3.2 and 3.3 we assume that the monomer concentration is constant, $c_{1}(t) \equiv c_{1}$.

Ideally, the aim is to solve explicitly the system of ordinary differential equations (3.3)-(3.4) for the concentrations $\left\{c_{r}(t)\right\}_{r=2}^{\infty}$. However, for many physically relevant aggregation and fragmentation rates, such goals are wildly optimistic. Being more realistic, we aim to understand the long-term behaviour of solutions to the system, and answer questions such as 'is there convergence to an equilibrium or a steady-state solution?', 'can gelation occur?', 'can anything else occur (eg a periodic solution)?'. Mathematically, the terms 'steady-state' and 'equilibrium' are often treated as synonymous; however, physically it is useful to make a distinction. We will take 'equilibrium' to refer to the situation where the concentrations $c_{r}$ are such that the chemical reaction (3.1) is balanced, that is, the forward reaction occurs at the same rate as the reverse; and a 'steady-state' to be when all the time-derivatives in (3.3) vanish. As we shall see in the next section, these lead to different conditions on the concentrations $c_{r}$.

In the remainder of this section we first define properties of the system of equations (3.3)-(3.4) which arise naturally in applications. These are also useful for proving existence and uniqueness of solutions, for constructing exact solutions (as in Brilliantov \& Krapivsky [46] and King \& Wattis [47]), and 
assessing the accuracy of approximate solutions. Approximate solutions of a simple Becker-Döring system are then given to illustrate the type of kinetic behaviour observed in Becker-Döring systems with constant monomer concentration. We then describe the constant mass formulation of the Becker-Döring equations and describe the solution approached in the large-time limit (Sections 3.4 and 3.5). We show that many systems have a unique equilibrium solution, which is approached in the large-time limit. However, we also show that other systems have no such equilibrium solution and, in the large time limit, exhibit weak convergence to a solution which has a mass different to the initial mass of the system. Finally (in Section 3.6), we discuss metastable behaviour observed in the large-time kinetics of such systems. Metastability is the ability of a non-equilibrium state to persist for a long time.

\subsection{Properties}

Since each cluster $C_{r}$ is comprised of $r$ monomers, the contribution to the mass of the system as a whole from such clusters is $r c_{r}$, and the total mass in the system is often denoted $\varrho(t)=M_{1}(t)$, as defined by $(2.4)$.

At equilibrium, the forward component of the reaction (3.1) exactly balances the reverse rate for all cluster sizes $r$, that is, $a_{r} c_{r} c_{1}=b_{r+1} c_{r+1}$. Hence at equilibrium, all the fluxes $J_{r}$ are zero. The equilibrium concentrations $c_{\mathrm{eq}, r}$ can be written

$$
c_{\mathrm{eq}, r}=\frac{a_{r-1}}{b_{r}} c_{\mathrm{eq}, 1} c_{\mathrm{eq}, r-1}=\ldots=\frac{a_{r-1} \ldots a_{1}}{b_{r} \ldots b_{2}} c_{\mathrm{eq}, 1}^{r}=Q_{r} c_{\mathrm{eq}, 1}^{r} .
$$

The quantity $Q_{r}$ satisfies

$$
Q_{1}=1 \quad \text { and } \quad a_{r} Q_{r}=b_{r+1} Q_{r+1}
$$

$Q_{r}$ is known as the partition function (of (3.3)-(3.4)). The reason for this is that it can be related to the free energy via $Q_{r}=\exp \left(-G_{r} / k T\right)$, where $G_{r}$ is the Gibbs free energy of a cluster of size $r$ [48,49], $k$ is Boltzmann's constant $\left(1.38 \times 10^{-23} \mathrm{JK}^{-1}\right)$ and $T$ is the absolute temperature.

The term steady-state refers to a solution in which the time-derivatives in (3.3) are zero for all $r$. This is satisfied by the condition $J_{r}=J$ for some constant $J$ independent of both time and cluster size $(r)$. We thus have a oneparameter family of steady-state solutions, which includes the equilibrium solution as a special case (the case $J=0$ ). However, the term steady-state is a more general concept with a wider definition; there being steady-states 
which are not equilibria. Solving $J=J_{r}$ with (3.4) we find

$$
c_{\mathrm{sss}, r}=Q_{r} c_{1}^{r}\left(1-J \sum_{k=1}^{r-1} \frac{1}{a_{k} Q_{k} c_{1}^{k+1}}\right) .
$$

Since $J$ is arbitrary, these solutions form a one-parameter family, of which the equilibrium solution is a member. We claim that when the equilibrium solution diverges at large $r$, and a steady-state solution is convergent at large $r$ (or has a weaker singularity than the equilibrium solution), then the system approaches a steady-state rather than the equilibrium solution. We refer to this argument as the principle of least divergence. In Section 3.3.2 we give further justification of this result.

Let us assume that $a_{r}$ is non-decreasing with $r$, so that if $c_{r}$ diverges as $r \rightarrow$ $\infty$ then so does $a_{r} c_{r}$. We determine the value of $J$ which gives the weakest divergence in the limit $r \rightarrow \infty$ by requiring the term in brackets in (3.7) to vanish in the limit $r \rightarrow \infty$. Hence we obtain the expression for the flux of the least divergent steady-state size distribution, $J_{\mathrm{sss}}$, as

$$
J_{\mathrm{sss}}=1 / \sum_{k=1}^{\infty} \frac{1}{a_{k} Q_{k} c_{1}^{k+1}} .
$$

The result (3.8) enables (3.7) to be rewritten as

$$
c_{\mathrm{sss}, r}=c_{\mathrm{eq}, r} \sum_{k=r}^{\infty} \frac{1}{a_{k} c_{1} c_{\mathrm{eq}, k}} / \sum_{k=1}^{\infty} \frac{1}{a_{k} c_{1} c_{\mathrm{eq}, k}} .
$$

If $J_{\mathrm{sss}}$ is non-zero, $c_{\mathrm{sss}, r}$ has a less severe divergence as $r \rightarrow \infty$ than the equilibrium solution since $c_{\mathrm{sss}, r} / c_{\mathrm{eq}, r} \rightarrow 0$ in the large $r$ limit. Examples of equilibrium and steady-state solutions are given in Section 3.3.2 and illustrated in Figure 3.

The quantity $V$, defined by

$$
V\left(\left\{c_{r}\right\}\right)=\sum_{r=1}^{\infty} c_{r}\left(\log \left(\frac{c_{r}}{Q_{r} c_{1}^{r}}\right)-1\right),
$$

is related to the Helmholtz free energy by $F=k T \tilde{V} V$, under conditions of constant temperature $(T)$ and volume $(\tilde{V})[49,50]$. The function $V$ satisfies $\dot{V} \leq 0$, with equality only for the equilibrium solution $c_{r}=Q_{r} c_{1}^{r}$. For all time-dependent solutions $\dot{V}<0$. Thus, provided $V$ is bounded below, it is a Lyapunov function for (3.3)-(3.4). In Sections 3.3.3, 3.3.4 and 3.3.5 we will see one example where $V$ is bounded below and two where it is not. 
In proving relationships such as $\dot{V} \leq 0$, it is useful first to derive the identity

$$
\sum_{r=1}^{\infty} g_{r} \frac{\mathrm{d} c_{r}}{\mathrm{~d} t}=g_{1} J_{1}+\sum_{r=1}^{\infty}\left(g_{r+1}-g_{r}\right) J_{r}
$$

for sequences $\left\{g_{r}\right\}_{r=1}^{\infty}$ with $g_{r} \in \mathbb{R}$. It follows that

$$
\frac{\mathrm{d} V}{\mathrm{~d} t}=\sum_{r=1}^{\infty}\left(a_{r} c_{r} c_{1}-b_{r+1} c_{r+1}\right)\left[\log \left(b_{r+1} c_{r+1}\right)-\log \left(a_{r} c_{r} c_{1}\right)\right] \leq 0
$$

since, as $\log$ is a monotonic function, $(A-B)(\log A-\log B) \geq 0$.

\subsection{Example of Becker-Döring kinetics}

\subsubsection{Preliminaries}

In order to illustrate some of the kinetic features observed in a constant monomer concentration Becker-Döring model, following [51], we will describe a system in which both the aggregation and fragmentation rates are sizeindependent, that is, $a_{r}=a$ and $b_{r}=b$,

$$
\frac{\mathrm{d} c_{r}}{\mathrm{~d} t}=a c_{1} c_{r-1}-b c_{r}-a c_{1} c_{r}+b c_{r+1}, \quad(r \geq 2)
$$

In this case the partition function and equilibrium solution are given by

$$
Q_{r}=\left(\frac{a}{b}\right)^{r-1}, \quad c_{\mathrm{eq}, r}=\left(\frac{a c_{1}}{b}\right)^{r-1} c_{1}:=\theta^{r-1} c_{1}
$$

where $\theta:=a c_{1} / b$ is a useful measure of the relative strength of aggregation to fragmentation. If $\theta<1$ fragmentation dominates aggregation, few large clusters are formed and the equilibrium solution (3.14) converges to zero as $r \rightarrow \infty$ with the total number and mass of clusters being given by

$$
M_{0}^{\mathrm{eq}}=\frac{c_{1}}{(1-\theta)}, \quad M_{1}^{\mathrm{eq}}=\frac{c_{1}}{(1-\theta)^{2}} .
$$

We now construct the one-parameter family of steady-state solutions. By applying (3.7) or by solving $a c_{1} c_{r}-b c_{r+1}=J$, we find

$$
c_{\mathrm{Sss}, r}=c_{1}\left[\theta^{r-1}-\frac{J\left(\theta^{r}-\theta\right)}{a c_{1}^{2}(\theta-1)}\right]=\left(c_{1}-\frac{J}{b(\theta-1)}\right) \theta^{r-1}+\frac{J}{b(\theta-1)} .
$$


For $\theta<1$, we see that if $J>0$ the concentrations $c_{\mathrm{sss}, r}$ become negative at large $r$; and if $J<0$ the concentrations remain bounded away from $c_{\mathrm{sss}, r}=0$ as $r \rightarrow \infty$, leading to a solution with divergent mass. For $\theta>1$, there is in (3.16) a term of $O\left(\theta^{r-1}\right)$ as $r \rightarrow \infty$, which leads to a divergent distribution (and possibly negative concentrations).

\subsubsection{Large-time solution}

For $\theta<1$ and monodisperse initial data, that is, of the form $c_{r}(0)=0$ for all $r \geq 2$, the Lyapunov function (3.10) takes the value $V=V_{0}=-c_{1}$. By (3.12) we have $\dot{V} \leq 0$ with equality only when the system is at equilibrium. The equilibrium solution (3.14) implies $V$ takes the value $V=V_{\text {eq }}=-c_{1} /(1-\theta)<$ $V_{0}$. For $\theta<1$, starting from monodisperse initial data, the system will converge to the equilibrium solution.

For $\theta>1$, the equilibrium solution (3.14), which corresponds to $J=0$, diverges as $r \rightarrow \infty$. Inserting the equilibrium solution into the function $V$ we find $V=-M_{0}=-\sum_{r=1}^{\infty} \theta^{r-1} c_{1}$ which has no lower bound. Hence we consider the possibility of the system converging to a steady-state instead of the equilibrium solution.

If $J<b c_{1}(\theta-1)$ the steady-state solution (3.16) diverges in the large $r$ limit, in the same manner as the equilibrium solution. If $J>b c_{1}(\theta-1)$ then at large enough $r$ (namely $\left.r>1+\log \left(J /\left[J-b c_{1}(\theta-1)\right]\right) / \log (\theta)\right)$ the concentrations $c_{\mathrm{sss}, r}$ are negative. However, if $J=b c_{1}(\theta-1)$ the concentrations $c_{\mathrm{sss}, r}$ are given by $c_{\mathrm{sss}, r}=c_{1}$. Although this solution does not decay to zero as $r \rightarrow \infty$, the concentrations do not diverge in this limit either; and we have $c_{\mathrm{sss}, r}>0$ for all $r$. We claim that when $\theta>1$ the solution approached in the large-time limit is this steady-state $c_{\mathrm{sss}, r}=c_{1}$, which has flux $J_{\mathrm{sss}}=c_{1}\left(a c_{1}-b\right)$. Unfortunately this claim cannot be justified by direct use of the Lyapunov function (3.10), since $V$ is not bounded below when $\theta \geq 1$.

There is an alternative method of justifying our claim that monodisperse initial data will evolve to the steady state $c_{\mathrm{sss}, r}=c_{1}$. By use of an equivalence transform (see Appendix B of [51]), a system with $\theta>1$ can be mapped to a 'dual' system, under which this steady-state is mapped to an equilibrium solution. We summarise the transformation for the special case of size-independent rate coefficients (3.13) which we are considering this section, although the equivalence transform works for more general rate coefficients as well. Under the transformation $\widehat{c}_{r}(t)=c_{r}(t) / c_{1} c_{\mathrm{eq}, r}$ where $c_{\mathrm{eq}, r}$ is given by (3.14), the governing equations (3.13) become

$$
\frac{\mathrm{d} \widehat{c}_{r}}{\mathrm{~d} t}=b \widehat{c}_{r-1}-a c_{1} \widehat{c}_{r}-b \widehat{c}_{r}+a c_{1} \widehat{c}_{r+1}
$$


together with $\widehat{c}_{1}=1 / c_{1}$. This system can be written as $\dot{\widehat{c}}_{r}=\widehat{J}_{r-1}-\widehat{J}_{r}$ where $\widehat{J}_{r}=b \widehat{c}_{r}-a c_{1} \widehat{J}_{r+1}$. Note that the transformed system is again a constant monomer concentration formulation of the Becker-Döring equations, with the forward and backward rates $a c_{1}$ and $b$ having been reversed. The dual system (3.17) thus has a $\theta$-value of $\widehat{\theta}=b / a c_{1}=1 / \theta$, and when $\theta>1, \widehat{\theta}<1$.

Transforming monodisperse initial conditions in the original system, we obtain monodisperse initial data in the dual system. The dual system (3.17) has an equilibrium solution $\widehat{c}_{\mathrm{eq}, r}=\widehat{\theta}^{r-1} \widehat{c}_{1}$ which corresponds to $\widehat{J}_{r}=0$ for all $r$. For $\widehat{\theta}<1$, the dual system (3.17) has a Lyapunov function

$$
\widehat{V}\left(\left\{\widehat{c}_{r}\right\}\right)=\sum_{r=1}^{\infty} \widehat{c}_{r}\left(\log \left(\frac{\widehat{c}_{r}}{\hat{\theta}^{r-1} \widehat{c}_{1}}\right)-1\right) .
$$

At $t=0$ we find $\widehat{V}=-\widehat{c}_{1}$. The function $\widehat{V}$ satisfies $\dot{\hat{V}}<0$ everywhere except for the equilibrium solution, where $\widehat{V}$ achieves its lower bound of $V\left(\left\{\widehat{c}_{\mathrm{eq}, r}\right\}\right)=$ $-\widehat{c}_{1} /(1-\widehat{\theta})$. Hence $\widehat{V}$ is a Lyapunov function and, as shown above for the untransformed system, monodisperse initial data will evolve to the equilibrium solution. Now the equilibrium solution of the dual system corresponds to the expected steady-state solution of the untransformed system; specifically $\widehat{c}_{\text {eq }, r}=$ $\widehat{\theta}^{r-1} \widehat{c}_{1}$ implies $c_{r}=c_{\mathrm{eq}, r} c_{1} \widehat{c}_{\mathrm{eq}, r}=\theta^{r-1} \widehat{c}_{1} c_{1} \theta^{r-1} c_{1}=c_{1}$. Thus, by transforming to the dual system, (in which $\hat{\theta}<1$ ), we have deduced that monodisperse initial data in the original system with $\theta>1$ will evolve to the steady-state solution $c_{\mathrm{sss}, r}=c_{1}$.

To summarise, for $\theta>1$, the steady-state solution $c_{\mathrm{sss}, r}=c_{1}$ is approached in the large time limit, and this has the steady nonzero flux $J_{\mathrm{sss}}=c_{1}\left(a c_{1}-b\right)$; for $\theta<1$ the equilibrium solution $c_{\mathrm{eq}, r}=\theta^{r-1} c_{1}$, which corresponds to $J_{r} \equiv 0$, is approached in the large time limit. This postulated principle of least divergence assumes that the initial conditions of the system have finite mass; systems with a divergent mass at $t=0$ may behave differently, as observed by Menon \& Pego $[28,29]$ (in the case of Smoluchowski aggregation kinetics). A system initiated with a 'fat tail', that is where the concentrations $c_{r}$ decay only algebraically with $r$ as $r \rightarrow \infty$ may maintain the slow decay and converge to a steady-state solution with steady flux $J_{\text {sss }}$ given by something other than (3.8).

In Figure 3 we plot $c_{\mathrm{sss}, r}$ both as a function of $r$ for certain fluxes $J$ (left-hand graph) and as a function $J$ for certain cluster sizes $r$ (right). In both cases we observe that for fluxes $J>J_{\text {sss }}$ the concentrations become negative at large cluster sizes - which is unphysical. For fluxes $J<J_{\text {sss }}$, the concentrations of larger cluster sizes diverge more rapidly than when $J=J_{\text {sss }}$.

The observant reader will have noticed that the above analysis has omitted the case $\theta=1$. A derivation similar to that shown above for $\theta>1$ can be 

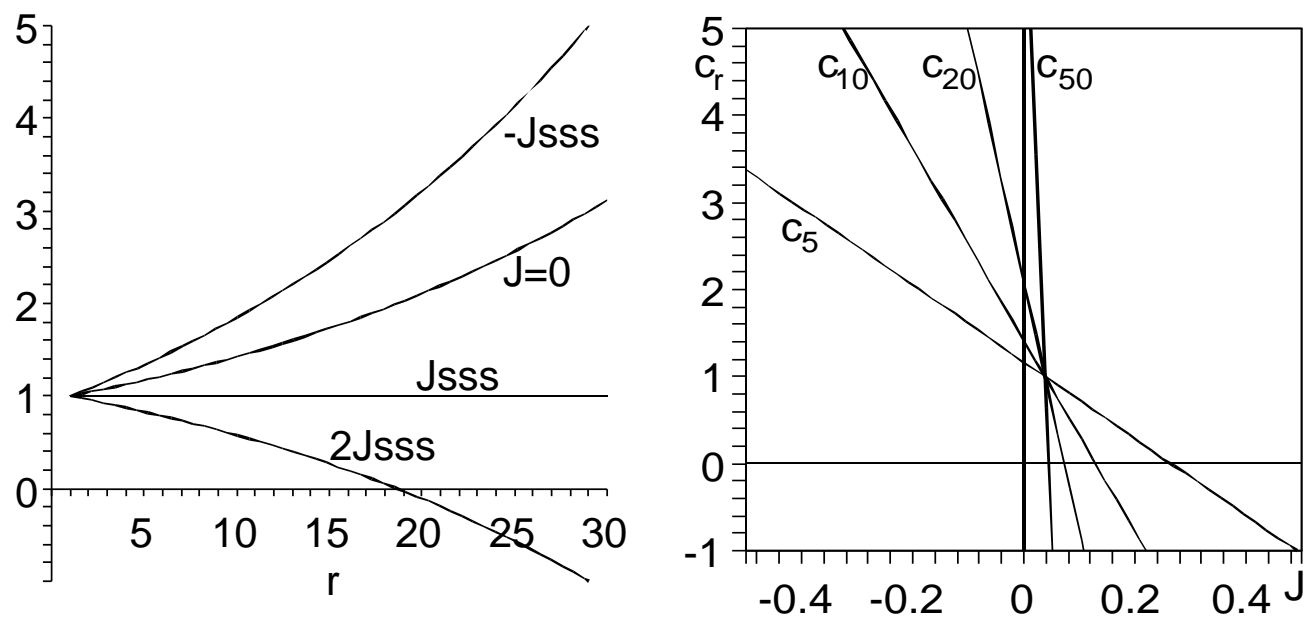

Fig. 3. Left: plot of the steady-state concentrations $c_{\mathrm{sss}, r}$ against $r$ for a variety of values of the flux parameter $J$ as given by (3.7). From uppermost curve: $J=-J_{\text {sss }}$, $J=0$ (the equilibrium solution), $J=J_{\mathrm{sss}}$ (for which $c_{\mathrm{sss}, r}=c_{1}$ ), and $J=2 J_{\mathrm{sss}}$. Right: plot of the steady-state values $c_{\mathrm{sss}, r}$ against $J$ for a variety of cluster sizes, as given by (3.7). From the most gentle to the steepest gradients, the curves correspond to $r=5, r=10, r=20$ and $r=50$. Parameter values: $\theta=1.04, a_{r}=1, c_{1}=1$, $b_{r}=1 / \theta$ so $J_{\mathrm{sss}}=0.03846$.

carried out for $\theta=1$. In place of (3.16), such a calculation leads to

$$
c_{\mathrm{sSs}, r}=c_{1}\left(1-\frac{J(r-1)}{b c_{1}}\right) .
$$

Minimising the strength of the divergence in the large $r$ limit leads to $J=0$, so this case approaches the equilibrium solution $c_{\mathrm{eq}, r}=c_{1}$. This equilibrium solution, however, has the same form as the steady-state solution when $\theta>1$, namely $c_{\mathrm{sss}, r}=c_{1}$.

The above arguments reveal which solution we expect the system to be attracted to in the large-time limit. However, all the solutions considered above are time-independent, and therefore we have learnt nothing about the transient behaviour and rate of approach to an equilibrium or a steady-state solution. This will be rectified in the following subsections where we investigate in more detail the solution in the large-time limit.

In what follows we obtain valuable information about the possible form of the solution of (3.13) at large times using a heuristic and non-rigorous technique of constructing an asymptotic approximation. In the particular example studied here, an exact solution in terms of an integral has been found, so Laplace's method and Watson's Lemma $[52,53]$ can be used to make the results of Sections 3.3.3-3.3.5 rigorous (as outlined in Section 3.3.6). However, for more general rate coefficients, the methodology described below is still applicable 
and generates useful results. The problem of rigorously establishing results derived using this methodology remains open.

\subsubsection{Case I: large-time kinetics for $\theta<1$}

In this case we expect the system to converge to the equilibrium solution $c_{\mathrm{eq}, r}=Q_{r} c_{1}^{r}$; to investigate how this happens we substitute $\psi_{r}(t)=c_{r}(t) / Q_{r} c_{1}^{r}$ so that $\psi_{r}(t) \rightarrow 1$ as $t \rightarrow \infty$. We thus find the equation for the evolution of $\psi_{r}(t)$ from $(3.3)-(3.4)$ as

$$
\frac{1}{b} \frac{\mathrm{d} \psi_{r}}{\mathrm{~d} t}=\psi_{r-1}-\psi_{r}-\theta \psi_{r}+\theta \psi_{r+1}, \quad(r \geq 2),
$$

in addition to $\psi_{1}=1$. If we think of $r$ as a spatial variable, then this becomes a boundary condition. We also assume that at $t=0$ there are no clusters of infinitely large size so that $\psi_{r}(0) \rightarrow 0$ as $r \rightarrow \infty$. Initial data which does not satisfy this condition may evolve in a manner different to that described below. Equation (3.20) is reminiscent of a diffusion equation in discrete space.

We generate an approximate solution for $\psi_{r}(t)$ based on the assumption that at large times $\psi_{r}$ varies only slowly in $r$; later we will verify this assumption. Hence we approximate the system (3.20) by taking the continuum limit in $r$, with $\Psi(r, t)=\psi_{r}(t)$, to obtain the equation

$$
\frac{1}{b} \frac{\partial \Psi}{\partial t}=\frac{1}{2}(1+\theta) \frac{\partial^{2} \Psi}{\partial r^{2}}-(1-\theta) \frac{\partial \Psi}{\partial r}
$$

which, as pointed out by Duncan \& Soheili [54], is in effect a Fokker-Planck equation. In deriving this equation, third, fourth, and higher $r$-derivatives of $\Psi(r, t)$ have been ignored. These terms can be retained in a higher order analysis and used to calculate correction terms. At leading order, we have the solution

$$
\Psi(r, t)=\frac{1}{2} \operatorname{erfc}\left(\frac{r-s(t)}{\sqrt{2 b(1+\theta) t}}\right)
$$

where $s(t)$ is the position of the wavefront and $\operatorname{erfc}(\cdot)$ is the complementary error function [31], which has the properties $\operatorname{erfc}(0)=1, \operatorname{erfc}(\eta) \rightarrow 0^{+}$as $\eta \rightarrow$ $\infty, \operatorname{erfc}(\eta) \rightarrow 2^{-}$as $\eta \rightarrow-\infty$, and so asymptotically satisfies the boundary conditions. We now determine the sizes of terms retained and neglected in the derivation of (3.21): at large times, the neglected terms $\Psi_{r r r}=O\left(t^{-3 / 2}\right)$ and $\Psi_{r r r}=O\left(t^{-2}\right)$ are much smaller than the retained terms $\Psi_{r}(r, t)=O\left(t^{-1 / 2}\right)$ 
and $\Psi_{r r}=O(1 / t)$. Since $s(t)$ is determined by $\mathrm{d} s / \mathrm{d} t=b(1-\theta)$, we have $s(t) \sim\left(b-a c_{1}\right) t$ as $t \rightarrow \infty$, and

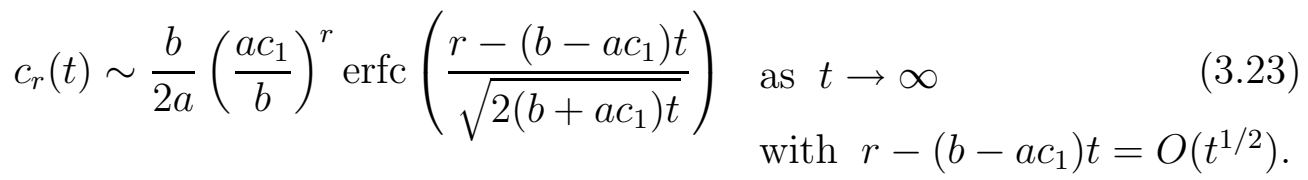

Such a situation is illustrated in Figure 4. The cluster distribution function has three regions: (i) $r / s(t)<1$, where the system has in effect already reached equilibrium $(1 \lesssim r \lesssim 60)$, (ii) the transition region $(70 \lesssim r \lesssim 100)$ given formally by $r / s(t)=1+O\left(t^{-1 / 2}\right)$ where $\psi$ is making the transition from its initial condition of $\psi=0$ to the equilibrium value of $\psi=1$, and (iii) $r / s(t)>1$ where the $c_{r}(t)$ are still asymptotically small $(110 \lesssim r)$, i.e. there is virtually no change from the initial condition. As $t$ increases the transition region moves to larger $r$ and widens. If one considers monodisperse initial data and is interested in the evolution of $c_{R}(t)$ for some large value of $R$, what one observes is a long induction time, of duration $t_{\text {ind }} \sim R /\left(b-a_{c} 1\right)$ during which $c_{R}(t)$ remains extremely small; then over a timescale of $t_{\text {trans }} \sim \sqrt{2\left(b+a c_{1}\right) R} /\left(b-a c_{1}\right)^{3 / 2}$, $c_{R}(t)$ evolves from almost zero to its equilibrium value, where it remains for all larger times.

As $t \rightarrow \infty$, the free energy $V$ approaches its lower bound, via the asymptotic behaviour

$$
V\left(\left\{c_{r}\right\}\right) \sim-\frac{c_{1}}{1-\theta}+\frac{c_{1} \theta^{\left(b-a c_{1}\right) t}}{1-\theta}
$$

and the total mass of the system (3.13), given by $M_{1}(t)=\sum_{r=1}^{\infty} r c_{\mathrm{eq}, r} \psi_{r}(t)$, is

$$
M_{1}(t) \sim \frac{c_{1}}{(1-\theta)^{2}}\left(1-b(1-\theta)^{2} t \mathrm{e}^{-b t(1-\theta) \log (1 / \theta)}\right)
$$

and thus can be seen to approach its equilibrium value (3.15).

\subsubsection{Case II: large-time kinetics for $\theta=1$}

In this case the equilibrium solution is $c_{\mathrm{eq}, r}=c_{1}$ and convergence is by a stationary diffusive wave which simply widens, according to

$$
c_{r}(t)=c_{1} \operatorname{erfc}\left(\frac{r}{2 \sqrt{b t}}\right) \text { as } t \rightarrow \infty \text { with } r=O\left(t^{1 / 2}\right) .
$$




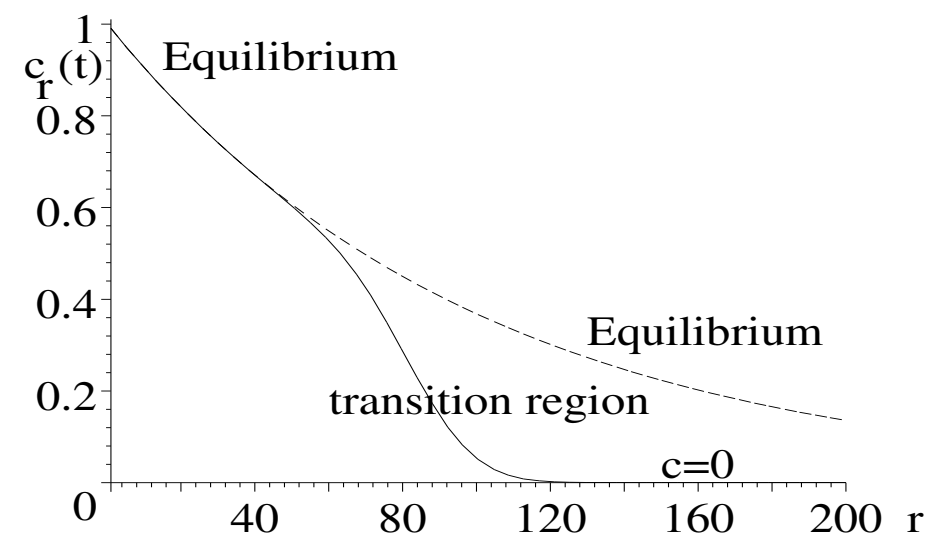

Fig. 4. Evolution of the case $\theta<1$ to equilibrium.

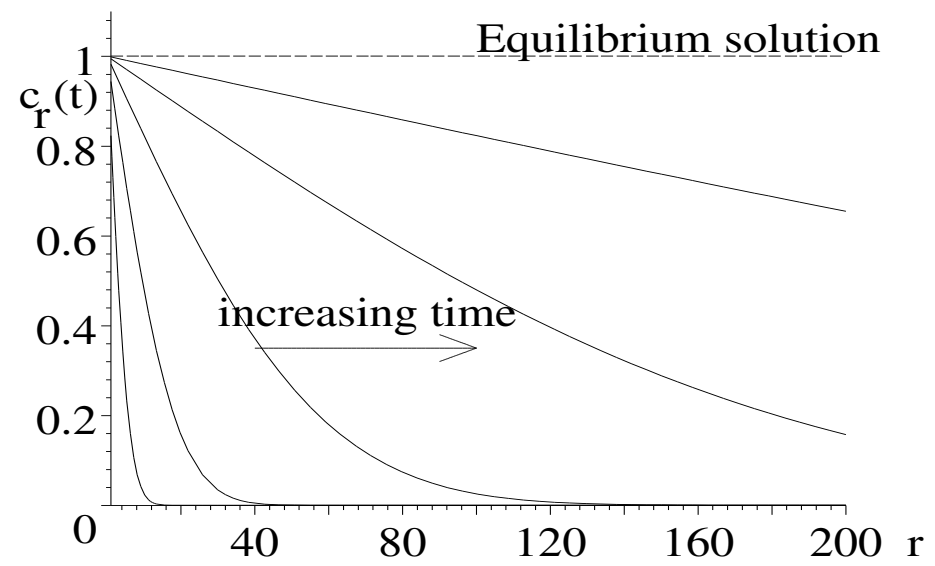

Fig. 5. Evolution of the case $\theta=1$ to equilibrium, the cluster size distribution is plotted at $t=10,10^{2}, 10^{3}, 10^{4}, 10^{5}$ when the parameters are given by $b=1, c_{1}=1$, $a=1$.

See Figure 5 for an illustration of this solution over the range $1 \leq r \leq 200$, $10 \leq t \leq 10^{4}$. In this case, at large times, the total mass of the system (3.13) grows according to $M_{1}(t) \sim b c_{1} t$, and the free energy follows

$$
V\left(\left\{c_{r}\right\}\right) \sim 2 c_{1} \sqrt{b t} \int_{0}^{\infty} \operatorname{erfc}(\eta)(\log (\operatorname{erfc}(\eta))-1) \mathrm{d} \eta
$$

which is negative ( $\operatorname{since} 0<\operatorname{erfc} \eta<1$ for $\eta>0$ ) and increases in magnitude without bound. Thus in this case the function $V\left(\left\{c_{r}\right\}\right)$ is not bounded below and so is not a Lyapunov function for the system.

\subsubsection{Case III: large-time kinetics for $\theta>1$}

In this case we put $\psi_{r}(t)=c_{r}(t) / c_{1}$ as the system converges to the steadystate solution $c_{\mathrm{sss}, r}=c_{1}$ (which has flux $J=J_{\mathrm{sss}}:=a c_{1}^{2}(1-1 / \theta)=c_{1}\left(a c_{1}-b\right)$ ) rather than the equilibrium solution $(J=0)$. The equilibrium solution $c_{\text {eq }, r}=$ $\theta^{r-1} c_{\text {eq, } 1}$ diverges at large $r$, as do all steady-state solutions with $J<J_{\text {sss }}$; 
steady solutions with fluxes $J>J_{\text {sss }}$ all lead to negative concentrations at large $r$.

The kinetic behaviour of $\psi_{r}(t)$ is similar to that of Case I; after taking the continuum limit in $r$, the partial differential equation for $\Psi(r, t)=\psi_{r}(t)$ is

$$
\frac{1}{b} \frac{\partial \Psi}{\partial t}=\frac{1}{2}(1+\theta) \frac{\partial^{2} \Psi}{\partial r^{2}}-(\theta-1) \frac{\partial \Psi}{\partial r} .
$$

Hence

$$
c_{r}(t) \sim \frac{1}{2} c_{1} \operatorname{erfc}\left(\frac{r-\left(a c_{1}-b\right) t}{\sqrt{2\left(a c_{1}+b\right) t}}\right) \quad \begin{aligned}
& \text { as } t \rightarrow \infty \\
& \text { with } r-\left(a c_{1}-b\right) t=O\left(t^{1 / 2}\right) .
\end{aligned}
$$

A typical solution is plotted in Figure 6. Starting from monodisperse initial data, the evolution of $c_{R}(t)$ for large $R$ is governed by a long induction time during which $c_{R}(t)$ remains small. This lasts for a time of $t_{\text {ind }} \sim R /\left(a c_{1}-b\right)$; there follows a transition phase of duration $t_{\text {trans }} \sim \sqrt{2\left(a c_{1}+b\right) R} /\left(a c_{1}-b\right)^{3 / 2}$ over which the concentration increases from almost zero to close to its steadystate value of $c_{1}$. Finally, over a longer timescale $c_{R}(t)$ converges to this steady value.

For the case $\theta>1$, the function $V\left(\left\{c_{r}\right\}\right)$ decreases according to

$$
V\left(\left\{c_{r}\right\}\right) \sim-\frac{1}{2} c_{1}\left(a c_{1}-b\right)^{2} \log \left(\frac{a c_{1}}{b}\right) t^{2} \quad \text { as } t \rightarrow \infty,
$$

thus $V$ is not bounded below and so does not qualify as a Lyapunov function. At large times, the leading-order approximation for the total mass of the system (3.13) is $M_{1}(t) \sim \frac{1}{2}\left(a c_{1}-b\right)^{2} c_{1} t^{2}$. Note that this represents a much faster rate of growth than that observed when $\theta=1$ (Case II, where $M_{1}(t) \sim a c_{1}^{2} t$ ). The free energy (3.30) also decreases much faster than (3.27). In both cases the difference is indicative of the fact that the case $\theta=1$ lies on the borderline between steady-state and equilibrium behaviour. In Case II aggregation and fragmentation are precisely balanced, whereas in Case III aggregation dominates.

\subsubsection{Alternative derivation of asymptotic results}

In [51] the exact solution of (3.13) for monodisperse initial data is given as

$$
c_{r}(t)=(r-1) c_{1} \theta^{(r-1) / 2} \int_{u=0}^{t} u^{-1} \mathrm{e}^{-\left(b+a c_{1}\right) u} I_{r-1}\left(2 u \sqrt{b a c_{1}}\right) \mathrm{d} u,
$$




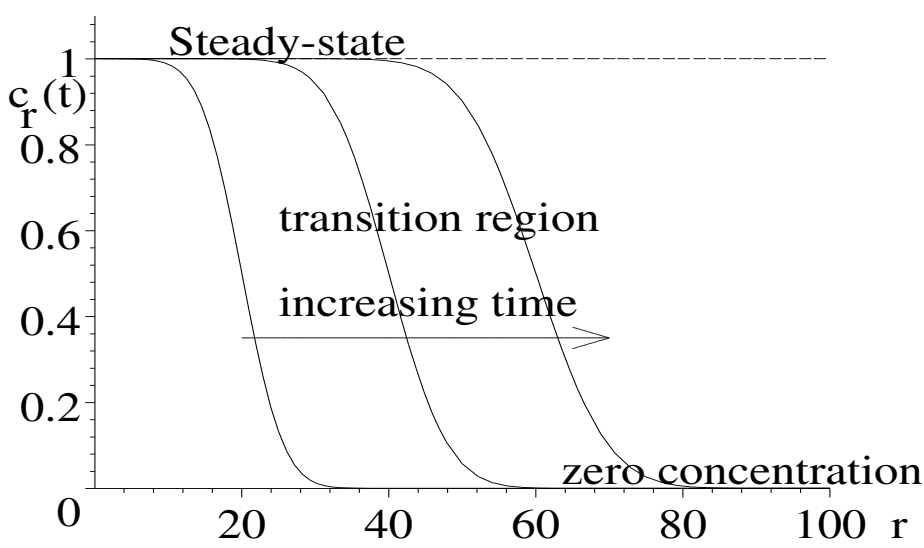

Fig. 6. Evolution of the case $\theta>1$ to steady-state, the cluster size distribution is plotted at $t=200,400,600$, for the parameters $b=0.9, c_{1}=1, a=1$, so $\theta=10 / 9$. The front propagates at a speed of 0.1 , so in the plots the front is at $r=s(t)=20,40,60$. Note that the width of the front grows as $t$ increases.

where $I_{\nu}(z)$ is the modified Bessel function [31]. The large-time kinetics of this solution can be approximated using equation 9.7 .7 on p.378 of [31], which allows the integral (3.31) to be rewritten as

$$
c_{r}(t) \sim \frac{c_{1} \theta^{\nu / 2} \sqrt{\nu}}{\sqrt{2 \pi}} \int_{z=0}^{2 b t \sqrt{\theta} / \nu} \frac{\mathrm{e}^{\nu f(z)} \mathrm{d} z}{z\left(1+z^{2}\right)^{1 / 4}}
$$

where $\nu=r-1=O(t) \gg 1$ and the function $f(z)$ is defined by

$$
f(z)=\sqrt{1+z^{2}}+\log z-\log \left(1+\sqrt{1+z^{2}}\right)-\frac{(\theta+1) z}{2 \sqrt{\theta}} .
$$

For simplicity, we now consider just the case $\theta>1$. In Laplace's method the function $f(z)$ is replaced by its Taylor expansion around either its maxima, or the endpoints of the interval $0 \leq z \leq 2 b t \sqrt{\theta} / \nu$. For large $t$, the relevant critical point is located at $z=z_{c}=2 \sqrt{\theta} /(\theta-1)$, where we find $f_{c}=f\left(z_{c}\right)=$ $-(\log \theta) / 2$ and $f_{c}^{\prime \prime}=f^{\prime \prime}\left(z_{c}\right)=-(\theta-1)^{3} / 4 \theta(\theta+1)$. At leading order, the integrand $g(z) \exp (\nu f(z))$ in (3.32) is replaced by $g\left(z_{c}\right) \exp \left(\nu f_{c}+\nu\left(z-z_{c}\right)^{2} f_{c}^{\prime \prime} / 2\right)$ which can be integrated explicitly. Ultimately one recovers the asymptotic result (3.29). For $\theta<1$, a similar calculation (based on an expansion around $\left.z_{c}=2 \sqrt{\theta} /(1-\theta)\right)$ reproduces $(3.23)$. At every approximation, the size of error terms in the calculation can be determined, hence it is possible to make this method rigorous. However, the analysis of this section relies on knowledge of the exact solution (3.31), which can be derived by taking the Laplace transform (in time) of (3.13). Exact solutions for more general rate coefficients are not known and not easily derivable. Whilst the heuristic method of Sections 3.3.3-3.3.5, is not rigorous, it is widely generalisable to rate coefficients which vary with cluster size (see, for instance, [47]). 


\subsection{Constant mass formulation of the Becker-Döring equations}

Some systems may be 'closed' in that only material present in the system at $t=0$ is available for growth of clusters, and there is no mechanism for replenishment of monomers from the outside. In such closed systems the monomer concentration will also vary, according to

$$
\frac{\mathrm{d} c_{1}}{\mathrm{~d} t}=-J_{1}(t)-\sum_{r=1}^{\infty} J_{r}(t)
$$

in addition to

$$
\begin{aligned}
\frac{\mathrm{d} c_{r}}{\mathrm{~d} t} & =J_{r-1}(t)-J_{r}(t) \quad(r \geq 2) \\
J_{r}(t) & =a_{r} c_{r}(t) c_{1}(t)-b_{r+1} c_{r+1}(t) .
\end{aligned}
$$

In this system of equations $c_{1}(t)$ also has to be treated as an unknown variable on par with rest of the $c_{r}(t), r \geq 2$. The problem is now much more complex, since the coupling of $c_{1}(t)$ with other functions $c_{r}(t)$ makes the equations (3.34)-(3.36) both nonlinear in the $c_{r}(t)$ variables and nonlocal in the dependent variable $r$. However, the system still possesses a Lyapunov function; in place of (3.10) we now have

$$
V\left(\left\{c_{r}\right\}\right)=\sum_{r=1}^{\infty} c_{r}\left(\log \left(\frac{c_{r}}{Q_{r}}\right)-1\right)
$$

This system of equations (3.34) was written down and studied by Penrose and Lebowitz in [49], and independently by Burton [55], and has since received much attention, both due to the difficulties in proving the existence of solutions [12] and because of its usefulness in modelling real systems such as micellisation kinetics $[3,4]$.

Ball, Carr \& Penrose [12] prove the existence of solutions of (3.35)-(3.36) under the conditions of $a_{r}=O(r)$ as $r \rightarrow \infty$ and $M_{1}(0)<\infty$. Solutions exist in the positive cone $X^{+}$of the Banach space $X$ where

$$
X=\left\{y=\left(y_{r}(t)\right)_{r=1}^{\infty}:\|y\|<\infty\right\}, \quad\|y\|:=\sum_{r=1}^{\infty} r\left|y_{r}(t)\right|
$$

and $X^{+}=\left\{y \in X: y_{r}(t) \geq 0 \forall r, t\right\}$. 


\subsection{Large-time behaviour}

Ball, Carr \& Penrose [12] investigated rigorously the existence, uniqueness and mass conservation of solutions of (3.34). The most interesting outcome of their study is a type of metastability which we shall now summarise. This behaviour occurs for certain combinations of aggregation and fragmentation rate coefficients. Based on the partition function induced by the rate constants, a classification into four types of system can be made.

Given an initial distribution of cluster sizes $c_{r}(0)=c_{r}^{o}$, we define the initial mass of the system by

$$
\varrho_{o}:=M_{1}(0)=\sum_{r=1}^{\infty} r c_{r}^{o}
$$

Next, from the aggregation and fragmentation rates $\left(a_{r}, b_{r}\right.$, respectively) we define the partition function $Q_{r}$ by (3.6) and then we consider the oneparameter family of potential equilibrium solutions, $c_{\mathrm{eq}, r}=Q_{r} c_{\mathrm{eq}, 1}^{r}$. We consider this family to be parameterised by the monomer concentration at equilibrium, which we define by $z=c_{\mathrm{eq}, 1}$. Each value of $z \in \mathbb{R}^{+}$generates a potential equilibrium solution $c_{\mathrm{eq}, r}(z)=Q_{r} z^{r}$. For each $z$ we thus define the total mass by

$$
\varrho_{\mathrm{eq}}(z)=\sum_{r=1}^{\infty} r c_{\mathrm{eq}, r}(z)=\sum_{r=1}^{\infty} r Q_{r} z^{r}
$$

Thus $\varrho_{\mathrm{eq}}$ is a function of $z$ defined in terms of a power series, and so it is natural to determine the radius of convergence of the series, $R$, which may be infinite, finite or zero. As the power series has no constant term, $\varrho_{\text {eq }}(0)=0$. All other coefficients of $z$ in the power series $\left(r Q_{r}\right)$ are positive and so $\varrho_{\text {eq }}(z)$ is a monotonically increasing function of $z$ in $z>0$.

Now, given any particular initial data $\left(c_{r}^{o}\right)$, the total mass of the equilibrium solution $\varrho_{\text {eq }}(z)$ should be the same as that of the initial data ( $\varrho_{o}$ given by (3.39)). Thus to determine precisely which equilibrium solution is approached in the large time limit (equivalently, which value of $z$ the monomer concentration assumes at equilibrium), it is natural to attempt to solve the equation $\varrho_{\mathrm{eq}}(z)=\varrho_{\mathrm{o}}$ for $z$, the equilibrium monomer concentration.

There are four scenarios which this procedure yields, each of which is described briefly below, together with examples of corresponding aggregation and fragmentation rates. These depend on the qualitative shape of the graph of $\varrho_{\text {eq }}(z)$ against $z$. The four cases are illustrated in Figure 7. In Cases III and 
IV metastable behaviour is observed; this will be discussed in more detail in Section 3.6.

\subsubsection{Case I: $R=\infty$.}

In this case the function $\varrho_{\text {eq }}(z)$ has an infinite radius of convergence, and so $0 \leq \varrho_{\mathrm{eq}}(z)<\infty$ for all $z$ in $0 \leq z<\infty$. Moreover, $\varrho_{\mathrm{eq}}(z) \rightarrow \infty$, as $z \rightarrow \infty$. Therefore, given any initial mass $\left(\varrho_{o} \geq 0\right)$, there is an equilibrium solution with the same total mass; that is, solving $\varrho_{\text {eq }}(z)=\varrho_{o}$ gives a unique solution, $z \in \mathbb{R}^{+}$. A large initial mass $\left(\varrho_{o} \gg 1\right)$ gives rise to a large equilibrium monomer concentration $(z \gg 1)$.

If we choose aggregation and fragmentation rates given by $a_{r}=1, b_{r}=(r+$ $1)^{2} / r$, (so that fragmentation dominates at large cluster sizes), from (3.6) the partition function is $Q_{r}=1 / r r$ !. The total mass of the equilibrium solution $c_{\mathrm{eq}, r}=Q_{r} z^{r}$ is

$$
\varrho_{\mathrm{eq}}(z)=\sum_{r=1}^{\infty} \frac{z^{r}}{r !}=\mathrm{e}^{z}-1 .
$$

Given any $\varrho_{o} \geq 0$ there is a unique solution $z=c_{\text {eq }, 1} \geq 0$ of the equation $\mathrm{e}^{z}-1=\varrho_{o}$, namely $z=\log \left(1+\varrho_{o}\right)$. In particular, if $\varrho_{o}=0$ then the equilibrium monomer concentration is given by $z=c_{\mathrm{eq}, 1}=0$; and as $\varrho_{o}$ increases, $c_{\mathrm{eq}, 1}$ also increases, with $z=c_{\mathrm{eq}, 1} \rightarrow \infty$ as $\varrho_{o} \rightarrow \infty$, as illustrated qualitatively in Case I of Figure 7.

\subsubsection{Case II: $0<R<\infty, \varrho_{\mathrm{eq}}(R)=\infty$.}

In this case the function $\varrho_{\text {eq }}(z)$ has a finite radius of convergence $R<\infty$, and as $z$ approaches $R$, the function $\varrho_{\text {eq }}(z)$ diverges; that is, as $z=c_{\text {eq, } 1} \rightarrow R^{-}$, $\varrho_{\mathrm{eq}}(z) \rightarrow+\infty$. For any initial mass $\left(\varrho_{o}\right)$ the equation $\varrho_{\mathrm{eq}}(z)=\varrho_{o}$ has a unique solution for $z$ in the range $0 \leq z<R$. Thus there is an equilibrium solution with the same total mass as the initial conditions. If one considers large initial masses, then we find that as $\varrho_{o} \rightarrow \infty, z \rightarrow R^{-}$; there is an upper limit on equilibrium monomer concentration.

As an example of this case we choose the rates of Section 3.3, namely $a_{r}=a$, $b_{r}=b$. Then $Q_{r}=(a / b)^{r-1}$ (by applying (3.6)). The Lyapunov function (3.37) implies that the system evolves to the equilibrium solution $c_{\mathrm{eq}, r}=Q_{r} z^{r}$, for some $z$; we find the total mass at equilibrium is

$$
\varrho_{\mathrm{eq}}(z)=\sum_{r=1}^{\infty} r(a z / b)^{r-1} z=\frac{z}{(1-a z / b)^{2}} .
$$



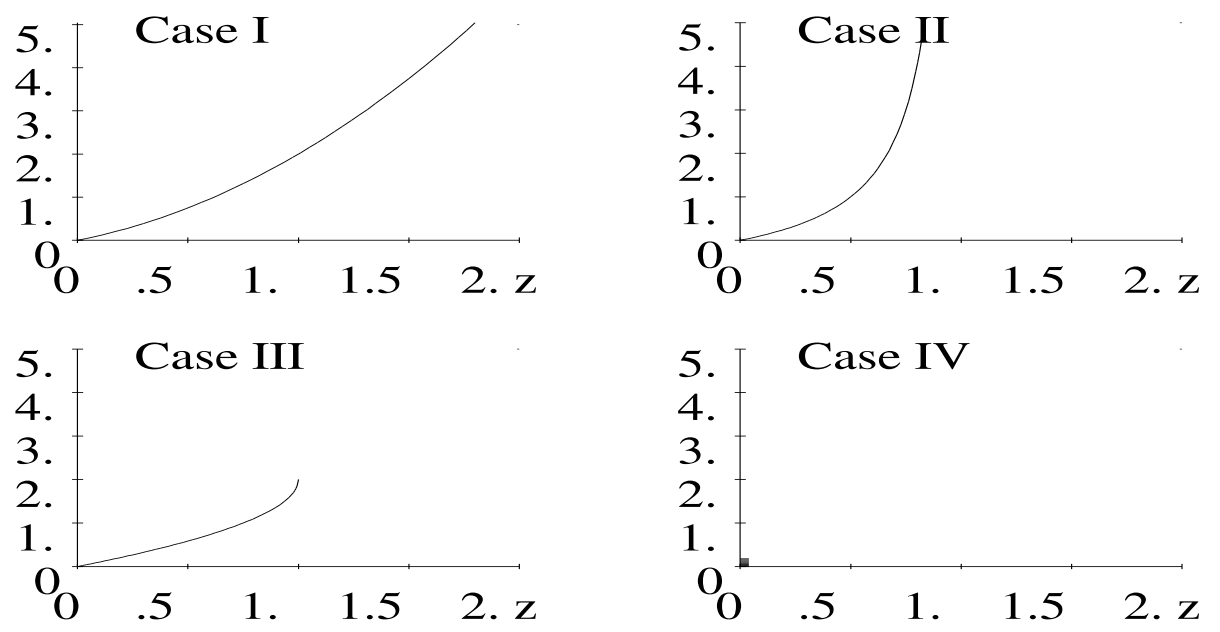

Fig. 7. The four cases for equilibrium data in the constant mass Becker-Döring system. In each case $\varrho_{\text {eq }}(z)$ is plotted against $z$. Case I corresponds to infinite radius of convergence; Case II, unit radius convergence, $R=1$ and $\varrho_{\text {eq }}(1)=\infty$; Case III, unit radius of convergence, $R=1$ and $\varrho_{\text {eq }}(1)=2$; and Case IV zero radius of convergence.

Given an initial mass of $\varrho_{o}$, the monomer concentration at equilibrium is given by solving the above for $z$, which leads to

$$
c_{\mathrm{eq}, 1}=z=\frac{2 \varrho_{o}}{1+2 a \varrho_{o} / b+\sqrt{1+4 a \varrho_{o} / b}}
$$

so $\varrho_{o}=0$ implies $z=0$ as expected, and as $\varrho_{o} \rightarrow \infty$ we have $z \rightarrow(b / a)^{-}$. Given any particular initial mass $\varrho_{o} \geq 0$, there is a unique equilibrium solution with the equilibrium monomer concentration lying in the range $0 \leq z<b / a$. No equilibrium solution can have a monomer concentration in the range $z \geq b / a$.

\subsubsection{Case III: $0<R<\infty$, $\varrho_{\mathrm{eq}}(R)<\infty$.}

In this case the function $\varrho_{\mathrm{eq}}(z)$ again has a finite radius of convergence, $R$, but, in contrast with Case II, when the monomer concentration takes on this maximum value, the total mass is finite. That is, at the radius of convergence $z=R$, the mass $\varrho_{\text {eq }}(z)$ takes on some maximum value, $\varrho_{c}:=\varrho_{\text {eq }}(R)<\infty$.

This case is not as simple as the two examples discussed above. If $\varrho_{o}<\varrho_{c}$, the equation $\varrho_{\text {eq }}(z)=\varrho_{o}$ has a unique solution and so, as in Case II, given such an initial mass, there is an equilibrium solution of the same mass which the system will approach in the large-time limit. However, if $\varrho_{o}>\varrho_{c}$ then there is no equilibrium solution with the same mass as the initial data. This prompts the question 'So what happens as $t \rightarrow \infty$ ?'

When $\varrho_{o}>\varrho_{c}$ we have an unusual type of convergence, with $c_{1}(t) \rightarrow R$ as 
$t \rightarrow \infty$ and hence $c_{r}(t) \rightarrow Q_{r} R^{r}$ also. However, the solution also maintains the initial mass, that is, the equation $\sum_{r=1}^{\infty} r c_{r}(t)=\varrho_{o}$ holds for all time $t$. The apparent anomaly that the mass of the limiting solution $\sum_{r=1}^{\infty} r Q_{r} R^{r}=$ $\varrho_{\text {eq }}(R)$ is strictly less than the initial mass, $\varrho_{o}$, is explained by the excess mass $\varrho_{o}-\varrho_{c}$ being transported to ever larger aggregation numbers. This has similar convergence properties to the solution of the diffusion equation $\left(u_{t}=u_{x x}\right)$ on an infinite line $x \in \mathbb{R}$. If the initial datum $u(x, 0)=\mathrm{e}^{-x^{2}}$ is imposed, then one expects the integral $I[u]=\int_{-\infty}^{\infty} u \mathrm{~d} x$ to be conserved $(I[u]=2 \sqrt{\pi}$ for all time). However, the solution $u(x, t)=\exp \left(-x^{2} / 4(t+1)\right) / \sqrt{t+1}$ shows that for each value of $x$ we have $u(x, t) \rightarrow 0$ as $t \rightarrow \infty$. Even though $I[0]=0$, the identity $I[u]=2 \sqrt{\pi}$ holds for every $t$. This means that the process of taking the limit $t \rightarrow \infty$ does not commute with evaluation of the integral $I[u]$. This property is known as 'weak' convergence; in contrast to that seen in Cases I and II, which is described as strong convergence.

If we are very careful in the choice of the aggregation and fragmentation rates, and take

$$
a_{r}=a, \quad b_{r+1}=\frac{b(r+1)^{2}}{r\left(r-\frac{1}{2}\right)},
$$

then

$$
Q_{r}=\left(\frac{a}{b}\right)^{r-1} \frac{\Gamma\left(r-\frac{1}{2}\right)}{r \sqrt{\pi} r !},
$$

by (3.6). The equilibrium solution $c_{\mathrm{eq}, r}=Q_{r} z^{r}$ implies that the mass at equilibrium depends on the equilibrium monomer concentration $\left(z=c_{\mathrm{eq}, 1}\right)$ according to

$$
\varrho_{\mathrm{eq}}(z)=\frac{2 z}{1+\sqrt{1-a z / b}} .
$$

This function is monotonically increasing from $z=0$ where $\varrho_{\text {eq }}(0)=0$ to $z=R:=b / a$ where $\varrho_{\mathrm{eq}}(R)=2 b / a$. Thus if $\varrho_{o}=0$ we have $z=c_{\mathrm{eq}, 1}=0$; and if we consider larger values of $\varrho_{o}$, then we are led to larger values of the solution $z=c_{\mathrm{eq}, 1}$; however, this only holds for initial masses $\varrho_{o}$ up to $\varrho_{c}=2 b / a$, where $z=c_{\text {eq }, 1}=b / a$. In the range $0 \leq \varrho_{o} \leq \varrho_{c}=2 b / a$, we have $0 \leq z \leq b / a$ and (3.46) can be inverted to give the equilibrium monomer concentration in terms of initial mass

$$
z=\varrho_{\mathrm{eq}}\left(1-\frac{a \varrho_{\mathrm{eq}}}{4 b}\right) \text {. }
$$

In Figure 7, III, we plot (3.46) which corresponds to the lower branch of 
(3.47), that is, $0 \leq \varrho_{\mathrm{eq}} \leq 2 b / a$. Whilst (3.47) is double-valued if $\varrho_{\text {eq }}$ is plotted against $z$, the single-valued solution (3.46) corresponds to the lower branch $\left(\varrho_{\text {eq }}<2 b / a\right)$, which is physically relevant. The upper branch of $(3.47)$, given by

$$
\widehat{\varrho}_{\mathrm{eq}}(z)=\frac{2 z}{1-\sqrt{1-a z / b}}
$$

gives rise to spurious solutions in which $2 b / a<\widehat{\varrho}_{\text {eq }} \leq 4 b / a$. Note that $z=0$, $\varrho_{o}=4 b / a$ solves $(3.47)$, and $\lim _{z \rightarrow 0} \widehat{\varrho}_{\mathrm{eq}}(z)=4 b / a$; however, substituting $z=0$ in (3.40) leads to $\varrho_{\mathrm{eq}}(0)=0$. It is thus $(3.46)$, which is the lower branch of (3.47) which leads to physically relevant solutions.

For initial masses $\varrho_{o}>\varrho_{c}$ there is no monomer concentration $c_{\mathrm{eq}, 1}=z$ which gives an equilibrium solution with mass $\varrho_{o}$ (that is, there is no solution $z$ to the problem $\left.\varrho_{\mathrm{eq}}(z)=\varrho_{o}\right)$. If a Becker-Döring system is started with mass $\varrho_{o}>\varrho_{c}$, the monomer concentration evolves according to $c_{1}(t) \rightarrow R=b / a$ as $t \rightarrow \infty$ and the system evolves towards the corresponding equilibrium solution, $c_{r}(t) \rightarrow Q_{r}(b / a)^{r}$. Bounds on the rate at which this occurs have been obtained by Penrose [56]. Note that for $z<b / a$, the equilibrium solution decays exponentially at large cluster sizes; but for $z=b / a$ the decay is only algebraic, with $c_{r} \sim b /\left(a \sqrt{\pi} r^{5 / 2}\right)$ as $r \rightarrow \infty$. Such a distribution still has finite total mass, which will be equal to $\varrho_{c}=2 b / a$. For systems with an initial excess of mass $\left(\varrho_{o}>\varrho_{c}\right)$, the excess mass $\left(\varrho_{o}-\varrho_{c}\right)$ is transported to ever larger aggregation numbers in such a way that mass is conserved $\left(\sum_{r=1}^{\infty} r c_{r}(t)=\varrho_{o}\right.$ for any $t<\infty)$. One way of thinking about this phenomena is that the excess mass is escaping to form a superparticle akin to gelation in the Smoluchowski model; however, since the Becker-Döring model has only cluster-monomer interactions, the kinetics is considerably slowed, and the gelation transition does not occur at any finite time.

Note that although the phenomena described here and in Case II are quite different, it is determined through subtle differences in the rate coefficients For large cluster sizes, the rates (3.44) can be approximated by

$$
a_{r}=a, \quad b_{r+1}=b\left(1+\frac{5}{2 r}\right)
$$

the leading order terms are thus identical to Case II, the difference being in the $O(1 / r)$ correction term in $b_{r+1}$. 


\subsubsection{Case IV: $R=0$.}

This is similar to Case III, with $R=0$ and $\varrho_{\text {eq }}(R)=0$. The only equilibrium solution in this case is $c_{\mathrm{eq}, r}=0$, and this is the solution which is approached in the large-time limit, that is for all $r, c_{r}(t) \rightarrow 0$ as $t \rightarrow \infty$. All mass is carried to larger and larger aggregation numbers such that at every time $t$ we have $\sum_{r=1}^{\infty} r c_{r}(t)=\varrho_{o}$. A rigorous analysis of this case has been carried out by Carr $\&$ Dunwell [57].

To show an example of this case we choose $a_{r}=a r, b_{r}=b$, so that aggregation dominates at larger cluster sizes. We find $Q_{r}=(a / b)^{r-1}(r-1)$ !. For an equilibrium solution of the form $c_{r}=Q_{r} z^{r}$, the total mass is

$$
\varrho_{\mathrm{eq}}(z)=\sum_{r=1}^{\infty}(a / b)^{r-1} r ! z^{r},
$$

which is a divergent power series, that is, the series diverges for any $z \neq 0$. Thus our assumption of the equilibrium solution being $c_{\mathrm{eq}, r}=Q_{r} z^{r}$ is false unless $z=c_{\mathrm{eq}, 1}=0$. So $c_{1}(t) \rightarrow 0$ and $c_{r}(t) \rightarrow 0$ for all $r$ as $t \rightarrow \infty$. However, for all $t<\infty$, the total mass of the system remains constant for all finite times $\left(\sum_{r=1}^{\infty} r c_{r}(t)=\varrho_{o}\right)$.

\subsection{Metastability}

In classical statistical physics, metastability is often defined in terms of a system being in a local energy minimum, thus a metastable state is locally stable (in that small fluctuations die away) and the system remains in the state for a long time (see Keller \& Cheng [58], for example). Eventually a large enough random fluctuation will occur to perturb the system into a region where it will be attracted to the global minimum energy configuration.

However, since our Becker-Döring models are purely deterministic (describing the averaged or expected behaviour of systems) it is not possible for us to calculate the waiting time for a 'large enough random fluctuation' to occur. Instead, by metastable, we shall mean a state which has a 'dormant instability'; that is, a state which is stable to most perturbations, but evolves slowly along a trajectory.

An example of metastable kinetics is the behaviour of the heat equation on a dumb-bell shaped region [59]. Over a relatively short time period each lobe of the dumb-bell evolves rapidly to a uniform temperature, though each lobe may have a different temperature. Over a much slower timescale, heat passes along the small neck joining the two lobes. This final phase of the dynamics 
can be described as metastable.

In [56], Penrose discusses metastable states and behaviour in relation to the constant mass formulation of the Becker-Döring system. Rigorous results on metastable behaviour in both the constant total mass and constant monomer concentration formulations of the Becker-Döring system are proven by Penrose in [60]. Although metastable states are characterised by extremely long lifetimes and very slow evolution, they are not equilibrium states; and they exist in systems which have no equilibrium configuration; that is, in Cases III and IV (Sections 3.5.3 and 3.5.4).

We assume that the rate coefficients $a_{r}, b_{r}$ are such that Case III applies, and that the critical monomer concentration $c_{1}=R$ corresponds to a critical mass of $\varrho_{c}$. For monomer concentrations $z=R+\varepsilon$ with $0<\varepsilon \ll 1$ there is no equilibrium solution since the sequence $r Q_{r} z^{r}$ diverges as $r \rightarrow \infty$. However, for small $\varepsilon$ the terms $r Q_{r} z^{r}$ diverge slowly. By saying ' $X$ is algebraically large (small)' we mean $X=O\left(\varepsilon^{n}\right)$ for some $n<0(n>0)$; and by 'exponentially large (small)', we mean $X \varepsilon^{n} \rightarrow \infty(0)$ as $\varepsilon \rightarrow 0$ for all $n>0(n<0)$.

It is helpful to split the cluster size distribution into two parts, according to cluster size, the smaller clusters being those with size $r \leq m$ and the larger ones having $r>m$. We define $m=m(z)$ by the value of $r$ which minimises $a_{r} Q_{r} z^{r}$. For $z<R$, the sequence $a_{r} Q_{r} z^{r+1}$ converges to zero, but for $z>R$ the sequence diverges; we are interested in values of $z$ just above $R$, for which $m(z)$ is large. Penrose proves that

$$
c_{r}(t)= \begin{cases}Q_{r} z^{r} J(z) \sum_{k=r}^{\infty} \frac{1}{a_{k} Q_{k} z^{k+1}}, & (r \leq m), \\ Q_{r} R^{r}, & (r>m),\end{cases}
$$

is a metastable state of the constant mass form of the Becker-Döring equations. The flux from the smaller to the larger parts of the distribution is given by $J^{*}=a_{m} Q_{m} z^{m+1}$, and the mass of the larger component is given by $M_{1}^{*}=\sum_{r=m+1}^{\infty} r Q_{r} R^{r}$. Provided certain reasonable conditions on $a_{r}, b_{r}$ and $Q_{r}$ are satisfied, and that $\varepsilon=z-R$ is small, it is possible to prove that $m(z)$ is algebraically large, $J^{*}$ is exponentially small, and $M_{1}^{*}$ is exponentially small. Furthermore, a system initiated from (3.51) maintains $\left|c_{r}(t)-c_{r}(0)\right|$ exponentially small for an algebraically large time $t$ whilst $\lim _{t \rightarrow \infty}\left|c_{r}(t)-c_{r}(0)\right|$ is not exponentially small; that is, the system is changing, but evolves on an exponentially long timescale. 


\subsection{Summary}

In this section we have given a brief introduction to the Becker-Döring equations. They arise in two main formulations, the original formulation with a fixed monomer concentration and, more recently, a system in which the total mass of the system is fixed, leading to a variable monomer concentration. We have shown how the large-time kinetics may be calculated using asymptotic techniques. In the constant mass formulation we have shown how the choices of aggregation and fragmentation rate coefficients give rise to qualitatively different large-time phenomena, with some systems converging to an equilibrium solution strongly and others weakly. Finally we have shown that weak convergence can lead to metastable kinetics. Many other formulations of the Becker-Döring equations have been proposed; for example, that of Dreyer \& Duderstadt [61], studied in more detail by Hermann et al. [62], uses an alternative interpretation of the available free energy of the system. More general systems are discussed in the next section.

\section{More general coagulation-fragmentation processes}

In this section we show how the Smoluchowski coagulation equations and the Becker-Döring equations have been generalised to form models of more general processes involving coagulation and fragmentation.

\subsection{Fragmentation}

First we generalise the simple stepwise fragmentation of the Becker-Döring model of reversible cluster growth to show how more general fragmentary processes can be modelled. The reverse process of (2.1) is binary fragmentation, which we may write as $C_{r} \rightarrow C_{s}+C_{r-s}$, where the only restriction is $1 \leq s \leq r-1$. If we assume that this process occurs at the rate $b_{s, r-s}=b_{r-s, s}$, we obtain the kinetic equation [63]

$$
\frac{\mathrm{d} c_{r}}{\mathrm{~d} t}=-\frac{1}{2} c_{r} \sum_{s=1}^{r-1} b_{s, r-s}+\sum_{s=1}^{\infty} b_{r, s} c_{r+s}
$$

Ball \& Carr [11] have shown that (4.1) has solutions of the form $c_{r}(t)=\mathrm{e}^{\lambda t} f_{r}$. Unfortunately such solutions do not conserve mass. For certain fragmentation kernels $b_{r, s}$, for example $b_{r, s}=(r+s)^{\beta}$ with $\beta>-1$, the solution has $\lambda>0$ and $0 \leq f_{r} \leq r^{-\beta-3}$ so mass is well-defined $\left(\sum_{r=1}^{\infty} r f_{r}<\infty\right)$ and increases 
due to a spurious influx from infinitely large particles. Intuitively, this can be thought of as the reverse process to gelation in the Smoluchowski coagulation equations.

In greater generality than (4.1), it is possible to treat multiple fragmentation, whereby when a cluster breaks up, many fragments are produced. Following Ziff [64], it is more natural in this case to write the rate constant as a product of $\gamma_{r}$, the rate at which a cluster of size $r$ fragments, and $\beta(r \mid m)$, the daughter distribution, which describes the number of fragments of size $m$ produced when a cluster of size $r$ fragments. In place of (4.1), we then have

$$
\frac{\mathrm{d} c_{r}}{\mathrm{~d} t}=-\gamma_{r} c_{r}+\sum_{s=1}^{\infty} \beta(r+s \mid r) \gamma_{r+s} c_{r+s}
$$

subject to the condition that $\sum_{m=1}^{r-1} m \beta(r \mid m)=r$.

\subsection{Smoluchowski coagulation-fragmentation}

In general coagulation-fragmentation processes, we may expect all the aggregation processes to be reversible as in

$$
C_{r}+C_{s} \rightleftharpoons C_{r+s}, \quad \text { forall } \quad r, s \geq 1
$$

For this brief discussion, we only consider binary fragmentation. We denote the aggregation rate in (4.3) by $a_{r, s}$ and the fragmentation rate by $b_{r, s}$, both of which must be symmetric, that is $a_{r, s}=a_{s, r}$ and $b_{r, s}=b_{s, r}$. We apply the law of mass action, defining $W_{r, s}$ to be the net flux from $c_{r}$ and $c_{s}$ to $c_{r+s}$, and obtain the evolution equations

$$
\frac{\mathrm{d} c_{r}}{\mathrm{~d} t}=\frac{1}{2} \sum_{s=1}^{r-1} W_{s, r-s}-\sum_{s=1}^{\infty} W_{r, s}, \quad W_{r, s}=a_{r, s} c_{r} c_{s}-b_{r, s} c_{r+s}
$$

with $\dot{c}_{1}=-\sum_{s=1}^{\infty} W_{1, s}$

The system of equations (4.4) has a number of properties similar to that of the Becker-Döring model. In many applications we will expect there to be a well-defined equilibrium solution which is determined by requiring all the fluxes to vanish, $\left(W_{r, s}=0\right)$. Provided the rate coefficients satisfy a consistency condition, known as detailed balancing, the solution has the same form as for the Becker-Döring equations, as we now show. Let us consider just the $W_{r, 1}$ 
fluxes. From the forward and reverse rates $a_{r, 1}$ and $b_{r, 1}$ we construct a partition function from $a_{r, 1} Q_{r}=b_{r, 1} Q_{r+1}$ and $Q_{1}=1$ in an identical fashion to (3.6). The equilibrium solution is then $c_{\mathrm{eq}, r}=Q_{r} c_{1}^{r}$. Since equilibrium is a global property of the system, when $c_{r}$ takes its equilibrium value all the fluxes $W_{r, s}$ for $s>1$ must also be zero. (If this were not the case, then $c_{r}=Q_{r} c_{1}^{r}$ is not the global equilibrium solution). Thus once all the rate coefficients $a_{r, s}$ and $b_{r, 1}$ are specified, the remaining $b_{r, s}$ for $s>1$ cannot be chosen freely; rather they must satisfy $a_{r, s} Q_{r} Q_{s}=b_{r, s} Q_{r+s}$.

Existence and uniqueness results for the coagulation-fragmentation equations have been derived by many authors. The results of Ball \& Carr [11] are restricted to kernels of the form $a_{r, s}<K(r+s)$ and $\sum_{s=1}^{\lfloor(r+1) / 2\rfloor} s^{1-\alpha} b_{s, r-s}<$ $K r^{1-\alpha}$. The former inequality prevents any potential problems with the aggregation kernel allowing gelation. They prove existence, uniqueness and mass conservation results, thus removing the possibility of fragmentation causing an increase of mass - a possibility noted in Section 4.1. Considering strong fragmentation, that is, $\sum_{s=1}^{\lfloor(r-1) / 2\rfloor} s^{m} b_{s, r-s} \geq K(m) r^{m+\gamma}$, Carr [65] has further proved that the quantity

$$
V\left(\left\{c_{r}\right\}\right)=\sum_{r=1}^{\infty} c_{r}(t)\left(\log \left(\frac{c_{r}(t)}{Q_{r}}\right)-1\right)
$$

is a Lyapunov function for the system (4.4). Starting from arbitrary initial data with finite mass, the system must then converge to the equilibrium solution $c_{\mathrm{eq}, r}=Q_{r} c_{1}^{r}$ with $c_{1}$ such that the total mass of the system at equilibrium matches that of the initial data. Carr \& da Costa [66] also consider non-gelling aggregation kernels, together with a weak fragmentation condition $\left(\sum_{s=1}^{\lfloor(r+1) / 2\rfloor} s b_{s, r-s} \leq K r\right)$, to prove results similar to the metastability results for the constant mass Becker-Döring system that we summarised in Section 3.4 .

Jeon [39] proved the occurrence of gelation in coagulation-fragmentation problems for which the aggregation kernel satisfies $\varepsilon r s<a_{r, s}<K r s$ in the limit $r+s \rightarrow \infty$ for some $0<\varepsilon \leq K<\infty$, and when the fragmentation kernel satisfies $(r+s) b_{r, s}<B$. The gelation time is bounded by $t_{g} \leq 4 /(2 \varepsilon \varrho-B)$ (provided $\varrho>C / \varepsilon$ ). Escobedo et al. [40] also prove the occurrence of gelation in systems with non-zero fragmentation terms.

One may expect the presence of a fragmentation term to alter the gelation behaviour of the system, allowing the mass to be conserved under different conditions to that of the pure coagulation equation (2.2). Da Costa [13] appears to be the first to prove that for aggregation kernels which permit delayed gelation in pure aggregation problems, namely $a_{r, s}$ satisfying only $a_{r, s}<K(r s)^{\alpha}$ with $\frac{1}{2}<\alpha<1$, mass-conserving solutions can exist provided that the fragmentation kernel is strong enough. The requirement on the frag- 
mentation kernel is that it is 'strong' in the terminology of Carr [65,66], namely $\sum_{s=1}^{r / 2} s^{\mu} b_{s, r-s} \geq K(\mu) r^{\mu+\gamma}$ for all $\mu \geq 1$ and for some $\gamma>0$.

\subsection{Multi-component coagulation-fragmentation}

Many cluster-growth problems inevitably involve the mixtures of different species forming heterogeneous clusters. This leads to multi-component problems, which have been formulated by a number of authors. For example, Wall \& Elvingson [67] and $\mathrm{Wu}$ [68] have analysed multi-component nucleation by way of generalised Becker-Döring-type models. Denoting the cluster composed of $r$ units of type A and $s$ units of type B by $c_{r, s}(t)$, the two-component BeckerDöring system is

$$
\frac{\mathrm{d} c_{r, s}}{\mathrm{~d} t}=J_{r-1, s}-J_{r, s}+I_{r, s-1}-I_{r, s}, \quad(r>1, s>1)
$$

together with

$$
J_{r, s}=a_{r, s} c_{r, s} c_{1,0}-b_{r+1, s} c_{r+1, s}, \quad I_{r, s}=\alpha_{r, s} c_{r, s} c_{0,1}-\beta_{r, s+1} c_{r, s+1}
$$

defined on the region $\Omega=\left\{(r, s) \in \mathbb{Z}^{2}: r \geq 0, s \geq\right.$, rs $\left.\neq 0\right\}$. The system (4.6) is subject to modified equations on $r=0$ and on $s=0$, namely $\dot{c}_{r, 0}=J_{r-1,0}-J_{r, 0}-I_{r, 0}(r \geq 2)$ and $\dot{c}_{0, s}=-J_{0, s}+I_{0, s-1}-I_{0, s}(s \geq 2)$. For the constant mass formulation, one naturally expects two conserved quantities, namely $M_{1,0}=\sum_{(r, s)} r c_{r, s}(t)$ and $M_{0,1}=\sum_{(r, s)} s c_{r, s}(t)$. Hence the above equations are complemented with $\dot{c}_{1,0}=-I_{1,0}-J_{1,0}-\sum_{\Omega} J_{r, s}$, and $\dot{c}_{0,1}=$ $-I_{0,1}-J_{0,1}-\sum_{\Omega} I_{r, s}$

There is an extra condition on the rate coefficients $a_{r, s}, b_{r, s}, \alpha_{r, s}, \beta_{r, s}$, which is similar to the detailed balancing condition observed above for the singlecomponent Smoluchowski coagulation-fragmentation equations. The equilibrium solution of (4.6)-(4.7) is obtained by requiring $J_{r, s}=0=I_{r, s}$ for all $(r, s)$, and so can be written as $c_{\mathrm{eq}, r, s}=Q_{r, s} c_{1,0}^{r} c_{0,1}^{s}$, where the partition function $Q_{r, s}$ satisfies

$$
a_{r, s} Q_{r, s}=b_{r+1, s} Q_{r+1, s}, \quad \text { and } \quad \alpha_{r, s} Q_{r, s}=\beta_{r, s+1} Q_{r, s+1}
$$

together with $Q_{1,0}=1=Q_{0,1}$. This implies that once $a_{r, s}, b_{r, s}$ and $Q_{0, s}$ are imposed then $Q_{r, s}$ can be determined. Knowledge of $\alpha_{0, s}$ and $\beta_{0, s}$ enables $Q_{0, s}$ to be found; specifying $\alpha_{r, s}$ for $s>0$ then completely specifies $\beta_{r, s}$ for $s>0$ (or vice versa). 
A multi-component Smoluchowski scheme has been proposed by Vemury et al. [69] for charged aerosols. Here, the quantity $c_{j, p}(t)$ denotes the concentration, at time $t$, of clusters of size (mass) $j$ with the second component $p$ being the charge of the cluster. In contrast to the mass parameter $j$, the charge parameter $p$ is allowed to take on negative values as well as positive. Thus equations such as

$$
\frac{\mathrm{d} c_{j, p}}{\mathrm{~d} t}=\frac{1}{2} \sum_{q=-\infty}^{\infty} \sum_{k=1}^{j-1} a_{k, q, j-k, p-q} c_{k, q} c_{j-k, p-q}-\sum_{q=-\infty}^{\infty} \sum_{k=1}^{\infty} a_{k, q, j, p} c_{k, q} c_{j, p},
$$

are derived. With these more complicated equations, the determination of the form of the rate coefficients $a_{k, q, j, p}$ also becomes more problematic. The size- and charge-dependencies of the aggregation coefficients all need to be postulated or determined before attempting to solve (4.9).

Another problem which ultimately leads to multi-component equations is cluster growth which depends upon both cluster size (mass) and shape (surface area or average diameter). Xiong \& Pratsinis [70], Kostoglou et al. [71] and Wattis [72] have studied such systems. Even the numerical solution of problems such as (4.9) is not straightforward since they require the repeated evaluation of double sums or double integrals, which is computationally costly, and difficult to do accurately particularly when the cluster distribution functions are sharply peaked. Hence a common approach is to make approximations so that the problems can be recast as single-component aggregation problems; see Vemury \& Pratsinis [73] for example.

\subsection{Summary}

In this section we have shown how generalisations of the Smoluchowski coagulation equations and the Becker-Döring equations have been formulated, including more general fragmentation kinetics. We have also noted several scenarios where multicomponent processes arise naturally, and illustrated how appropriate model equations may be constructed. Other interesting phenomena arise in the modelling of more complex physico-chemical cluster-growth problems include the inhibition of cluster growth by a second chemical species. Such processes lead to unusual kinetics, as studied in [74], where one approach to modelling the highly nonlinear process of secondary nucleation is proposed. Marques et al. [75,76] model the relaxation of worm-like polymers using combined coagulation-fragmentation processes such as end-interchange $\left(C_{j+k}+\right.$

$\left.C_{m} \rightarrow C_{j+m}+C_{k}\right)$ and bond-interchange $\left(C_{j+k}+C_{m+n} \rightarrow C_{j+m}+C_{k+n}\right)$, both of which conserve the total number of clusters as well as the total mass of clusters. 
There is a considerable literature relating the Lifshitz-Slyozov-Wagner $[77,78]$ theory for the growth of clusters at large times to Becker-Döring models of cluster growth, see Carr \& Penrose [79], Neithammer \& Pego [80,30] and Velazquez [81] for further details. Both Dubovski [82] and Laurençot [83,84] have studied the Oort-Hulst-Safronov (OHS) coagulation equation [85,86], the latter proving convergence to self-similar solutions in the continuous case. By constructing a more general discrete coagulation model, Dubovski demonstrates underlying connections between the OHS model and the Smoluchowski model.

Other effects which it would be desirable to include in models of aggregation processes include spatial effects such as diffusion, advection and fluid flow; for example, see Slemrod [16]. This area is complicated since typically diffusion rates decay to zero with increasing cluster size. An example of this is seen in models which assume only monomers are allowed to diffuse and all other cluster sizes are fixed. This conveniently leads to the Becker-Döring equations. Although such assumptions at first sight seem extreme, in certain cases they are justifiable - for example in Molecular Beam Epitaxy $[87,88]$ where clusters are two-dimensional islands with a thickness of one atomic layer on an otherwise perfectly flat surface: over the timescales of interest, single atoms are mobile, whereas dimers and trimers are, to a good approximation, stationary.

\section{Conclusions}

In this paper we have given a brief introduction to the Smoluchowski coagulation equation: in Section 2 we have shown how exact solutions may be derived for the three exactly solvable aggregation kernels, and summarised some of the more elementary self-similar scaling results. We have given an introduction to the phenomenon of gelation which occurs for certain classes of aggregation kernel, and have noted some of the existence results.

In Section 3 we have described some of the theory associated with the BeckerDöring model of stepwise aggregation and fragmentation. This system of equations is much simpler than the Smoluchowski system in that only clustermonomer interactions are permitted. Two versions of the system were discussed: firstly the constant monomer concentration formulation, for which we illustrated some simple features of the kinetics of cluster formation through the use of asymptotic expansions; secondly, the constant mass formulation, which leads to a more complicated mathematical problem (being both nonlinear and nonlocal), allowed us to discuss the phenomena of weak and strong convergence and illustrate the presence of metastable kinetics in the Becker-Döring model.

Finally, in Section 4 we have discussed some more sophisticated models which 
involve combinations and generalisations of coagulation and fragmentation processes. As well as solving the appropriate coagulation-fragmentation models, in any particular application there remains the challenge of determining appropriate rate coefficients.

A major current challenge is the understanding of coagulation of multi-component systems: this provides problems in the analysis of mass conservation and gelation properties. For example, in multicomponent systems there are many interesting problems surrounding gelation: how does the composition of the gel evolve over time ? can the two components gel separately at different times ? Also, the construction of existence and uniqueness results, the development of efficient numerical algorithms for computer simulation, and problems in the acquisition of vast arrays of data for the rate coefficients are all more complicated in multicomponent systems. Even in single-component coagulationfragmentation systems there are many results which have been derived by use of asymptotic methods but which not yet been rigorously established. Open problems include establishing the strength of fragmentation needed to prevent gelation in Smoluchowski systems. Other areas which have received little detailed mathematical analysis are systems with simultaneous input and removal of clusters, and systems which couple coagulation-fragmentation with spatial effects through advection-diffusion processes. The rigorous analysis of systems whose initial data is divergent (but possibly Borel summable) is an emerging area; this type of Smoluchowski system has recently been explored by Menon and Pego [28,29], but as yet there is no corresponding theory for Becker-Döring systems. In conclusion the area of coagulation-fragmentation problems remains open and active; the variety of applications from which model equations are derived, continually give rise to new systems of equations.

\section{Acknowledgements}

I would like to thank the American University of Beirut for inviting me to speak at the Workshop on Complex Systems in November 2002. An early version of this paper was prepared as part of a short lecture course for that meeting. I am deeply indebted to Michael Grinfeld for an extremely thorough reading of the manuscript and for making numerous suggestions for its improvement. I would also like to thank Oliver Penrose for helpful comments.

\section{References}

[1] HR Pruppacher \& JD Klett. Microphysics of Clouds and Precipitation. Reidel, Dordrecht, (1978). 
[2] RL Drake. In GM Hidy \& JR Brock (Eds) Topics in Current Aerosol Research, vol 3 pt 2. Pergamom Press, New York, (1972).

[3] SN Wall \& GEA Aniansson. Numerical calculations on the kinetics of stepwise micelle association. $J$ Phys Chem, 84, 727-736, (1980).

[4] PV Coveney \& JAD Wattis. Analysis of a generalized Becker-Doring model of self-reproducing micelles. Proc. Roy. Soc. Lond. A, 452, 2079-2102, (1996).

[5] WH Stockmayer. Theory of molecular size distribution and gel formation in branched-chain polymers. J Chem Phys, 11, 45-55, (1943).

[6] P Michel, E Benz, P Tanga \& DC Richardson. Collisions and gravitational reaccumulation: forming asteroid families and satellites. Science, 294, 16961700, (2001).

[7] MH Lee. On the validity of the coagulation equation and the nature of runaway growth. Icarus, 143, 74-86, (2000).

[8] M von Smoluchowski. Drei vorträge über diffusion Brownsche molekular bewegung und koagulation von kolloidteichen. Physik Z, 17, 557-571 (1916).

[9] R Becker \& W Döring. Kinetische behandlung der keimbildung in übersättigten dämpfen. Ann Phys, 24, 719-752, (1935).

[10] DJ Aldous. Deterministic and stochastic models for coalescence (aggregation, coagulation): review of the mean-field theory for probabilists. Bernouilli, 5, 3-48, (1999).

[11] JM Ball \& J Carr. The discrete coagulation-fragmentation equations: existence, uniqueness and density conservation. J Stat Phys, 61, 203-234, (1990).

[12] JM Ball, J Carr \& O Penrose. The Becker-Döring cluster equations: basic properties and asymptotic behaviour of solutions. Commun Math Phys, 104, 657-692, (1986).

[13] FP da Costa. Existence and uniqueness of density conserving solutions to the coagulation-fragmentation equations with strong fragmentation. J Math Anal Es Appl, 192, 892-914, (1995).

[14] JB McLeod. On an infinite set of nonlinear differential equations. $Q J$ Math Oxford (Ser 2), 13, 119-128, (1962).

[15] JB McLeod. On an infinite set of nonlinear differential equations (II). Q J Math Oxford (Ser 2), 13, 193-205, (1962).

[16] M Slemrod. Coagulation-diffusion systems: derivation and existence of solutions for the diffuse-interface structure equations. Physica D, 46, 351-366, (1990).

[17] WH White. A global existence theorem for Smoluchowski's coagulation equations. Proc Am Math Soc, 80, 273-276, (1980).

[18] JR Norris. Uniqueness, nonuniqueness and a hydrodynamic limit for the stochastic coalescent. Ann Appl Probab, 9, 78-109, (1999).

[19] PGJ van Dongen \& MH Ernst. Fluctuations in coagulating systems. J Stat Phys, 49, 879-926, (1987).

[20] PGJ van Dongen \& MH Ernst. Fluctuations in coagulating systems, II. J Stat Phys, 49, 927-975, (1987). 
[21] SC Davies, JR King \& JAD Wattis. Self-similar behaviour in the coagulation equations. J Eng Math, 36, 57-88, (1999).

[22] R Muraldir \& D Ramkrishna. An inverse problem in agglomeration kinetics. $J$ Colloid \& Interface Sci, 112, 348-361, (1986).

[23] DJ Smit, MJ Hounslow \& WR Paterson. Aggregation and gelation I analytical solution for CST and batch operation. Chem Eng Sci, 49, 1025-1035, (1994).

[24] FP da Costa. A finite dimensional dynamical model for gelation in coagulation processes. J Nonlinear Sci, 8, 619-653, (1998).

[25] JF Collet. Some modelling issues in the theory of fragmentation-coagulation systems. Comm Math Sci, 1, 35-54, (2004).

[26] M Kreer \& O Penrose. Proof of dynamic scaling in Smoluchowski's coagulation equation with constant kernels. J Stat Phys, 74, 389-407, (1994).

[27] FP da Costa. On the dynamic scaling behaviour of solutions to the discrete Smoluchowski equations. Proc Edinburgh Math Soc, 39, 547-559, (1996).

[28] G Menon \& RL Pego. Approach to self-similarity in Smoluchowski's coagulation equations. Commun Pure \& Appl Math, 57, 1197-1232, (2004).

[29] G Menon \& RL Pego. Dynamical scaling in Smoluchowski's coagulation equations: uniform convergence. SIAM J Math Anal, 36, 1629-1651, (2005).

[30] RL Pego. Lectures on dynamics in models of coarsening and coagulation. http://www. math. cmu . edu/ nw0z/publications/06-CNA-001/001abs/06-CNA-001.pdf

[31] M Abramowitz \& IA Stegun. Handbook of Mathematical Functions. Dover, New York, (1972).

[32] J Bertoin. Eternal solutions to Smoluchowski's coagulation equation with additive kernel and their probabilistic interpretations. Ann Appl Probab, 12, 547-564, (2002).

[33] RM Ziff \& G Stell Kinetics of polymer gelation. J Chem Phys, 73, 3492-3499, (1980).

[34] F Leyvraz \& HR Tschudi. Singularities in the kinetics of coagulation processes. J Phys A: Math Gen, 14, 3389-3403, (1981).

[35] F Leyvraz \& HR Tschudi. Critical kinetics near gelation. J Phys A; Math Gen, 15, 1951-1964, (1982).

[36] PGJ van Dongen. On the possible occurrence of instantaneous gelation in Smoluchowski's coagulation equation. J Phys A; Math Gen, 20, 1889-1904, (1987).

[37] MH Lee. A survey of numerical solutions to the coagulation equation. JPhys A Math Gen, 34, 10219-10242, (2001).

[38] J Carr \& FP da Costa. Instantaneous gelation in coagulation dynamics. $Z$ Angew Math Phys, 43, 974-983, (1992).

[39] I Jeon. Existence of gelling solutions for coagulation-fragmentation equations. Comm Math Phys, 194, 541-567, (1998).

[40] M Escobedo, S Mischler, B Perthame. Gelation in coagulation and fragmentation models. Comm Math Phys, 231, 157-188, (2002). 
[41] F Leyvraz. Scaling theory for gelling systems: work in progress. to appear in Physica D (this issue), ,-, (2006).

[42] H van Roessel \& M Shirvani. A formula for the post-gelation mass of a coagulation equation with a separable bilinear kernel. to appear in Physica $D$ (this issue), ,-, (2006).

[43] N Fournier \& P Laurençot. Existence of self-similar solutions to Smoluchowski's coagulation equation. Comm Math Phys, 256, 589-609, (2005).

[44] EM Hendricks, MH Ernst \& RM Ziff. Coagulation equations with gelation. $J$ Stat Phys, 31, 519-563, (1983).

[45] F Leyvraz. Scaling theory and exactly solved models in the kinetics of irreversible aggregation. Phys Rep, 383, 95-212, (2003).

[46] NV Brilliantov \& PL Krapivsky. Non-scaling and source-induced scaling behaviour in aggregation models of movable monomers and immovable clusters. J Phys A; Math Gen, 24, 4787-4803, (1991).

[47] JR King \& JAD Wattis. Asymptotic solutions of the Becker-Döring equations with size-dependent rate constants. J Phys A Math Gen, 35, 1357-1380, (2002).

[48] B Lewis, in Crystal Growth, ed BR Pamplin, pp12-39, Pergamon Press, Oxford, (1975).

[49] O Penrose \& JL Lebowitz. In "Studies in Statistical Mechanics VII: Fluctuation Phenomena", edited E. Montroll \& J.L. Lebowitz, pp.322-375. North Holland, Amsterdam, (1976).

[50] CJ Tompson. Classical Equilibrium Statistical Mechanics. OUP, Oxford, (1988).

[51] JAD Wattis \& JR King. Asymptotic solutions of the Becker-Döring equations. J Phys A; Math Gen, 31, 7169-7189, (1998).

[52] JD Murray. Asymptotic Analysis. Springer, Oxford, (1974).

[53] CM Bender \& SA Orszag. Advanced Mathematical Methods for Scientists and Engineers. McGraw-Hill, Singapore, (1978).

[54] DB Duncan \& AR Soheili. Approximating the Becker-Döring cluster equations. Appl Num Math, 37, 1-29, (2001).

[55] JJ Burton. Nucleation theory, pp195-234 of BJ Berne, Statistical Mechanics, Part A, Equilibrium techniques, Plenum, (1977).

[56] O Penrose. Metastable decay rates, asymptotic expansions and analytic continuation of thermodynamic functions. J Stat Phys, 78, 267-283, (1995).

[57] J Carr \& RM Dunwell. Asymptotic behaviour of solutions to the Becker-Doring equations. Proc Ed Math Soc, 42, 415-424, (1992).

[58] A Keller \& SZD Cheng. The role of metastability in polymer phase transitions. Polymer, 39, 4461-4487, (1998)

[59] J Carr. Personal communication, (1992).

[60] O Penrose. Metastable states for the Becker-Döring cluster equations. Commun Math Phys, 124, 515-541, (1989).

[61] W Dreyer \& F Duderstadt. On the Becker/Döring theory of nucleation of liquid droplets in solids. J Stat Phys, 123, 55-87, (2006). 
[62] M Hermann, M Naldzhieva \& B Niethammer. On a thermodynamically consistent modification of the Becker-Döring equations. To appear in Physica $D$ (this issue), ,-, (2006).

[63] R Simha. Kinetics of degradation and size distribution of long chain polymers. J Appl Phys, 12, 569-578, (1941).

[64] RM Ziff. An explicit solution to a discrete fragmentation model. J Phys A; Math Gen, 25, 2569-2576, (1992).

[65] J Carr. Asymptotic behaviour of solutions to the coagulation-fragmentation equations. I. The strong fragmentation case. Proc Roy Soc Ed, 121A, 231-244, (1992).

[66] J Carr \& FP da Costa. Asymptotic behaviour of solutions to the coagulationfragmentation equations. II. Weak fragmentation. J Stat Phys, 77, 89-123, (1994).

[67] S Wall \& C Elvingson. Equilibrium and kinetic properties of mixed micelles. $J$ Phys Chem, 89, 2695-2705, (1985).

[68] DT Wu. General approach to barrier crossing in multi-component nucleation. J Chem Phys, 99, 1990-2000, (1993).

[69] S Vemury, C Jansen \& SE Pratsinis. Coagulation of symmetric and asymmetric bipolar aerosols. J Aerosol Sci, 28, 599-611, (1997).

[70] Y Xiong \& SE Pratsinis. Formation of agglomerate particles by coagulation and sintering: part I. a two-dimensional solution to the population balance equation. J Aerosol Sci, 24, 283-300, (1993).

[71] M Kostoglou, AG Konstandopoulos \& SK Friedlander. Bivariate population dynamics simulation of fractal aerosol aggregate coagulation and restructuring. to appear in Aerosol Sci, 37, -, (2006). [doi:10.1016/j.jaerosci.2005.11.009]

[72] JAD Wattis. Exact solutions for cluster-growth kinetics with evolving size and shape profiles. J Phys A; Math Gen, 39, 7283-7298, (2006).

[73] S Vemury \& SE Pratsinis. Self-preserving size distributions of agglomerates. $J$ Aerosol Sci, 26, 175-185, (1995).

[74] JAD Wattis \& PV Coveney. Generalised nucleation theory with inhibition for chemically reacting systems. J Chem Phys, 106, 9122-9140, (1997).

[75] CM Marques, MS Turner \& ME Cates. End-evaporation kinetics in livingpolymer systems. J Chem Phys, 99, 7260-7266, (1993).

[76] CM Marques, MS Turner \& ME Cates. Relaxation mechanisms in worm-like micelles. J Non-Crystalline Solids, 172-174, 1168-1172, (1994).

[77] IM Lifshitz \& VV Slyozov. Kinetics of a precipitation from supersaturated solid solutions. J Phys Chem Sol, 19, 35-50, (1961).

[78] C Wagner. Theorie der alterung von niederschlägen durch umlösen. $Z$ Elektrochem, 65, 581-591, (1961).

[79] J Carr \& O Penrose. Asymptotic behaviour of solutions to a simplified LifshitzSlyozov equation. Physica D, 124, 166-176, (1998). 
[80] B Neithammer \& RL Pego. Non-self-similar behavior in the LSW theory of Ostwald ripening. J Stat Phys, 95, 867-902, (1999).

[81] JJL Velazquez. The Becker-Döring equations and the Lifshitz-Slyozov theory of coarsening. J Stat Phys, 92, 195-236, (1998).

[82] PB Dubovski. A triangle of coagulation models. J Phys A; Math Gen, 32, 781793, (1999).

[83] P Laurencot. Convergence to self-similar solutions for a coagulation equation. ZAMP, 56, 398-411, (2005).

[84] $\mathrm{Ph}$. Laurençot. Self-similar solutions to a coagulation equation with multiplicative kernel. To appear in Physica D (this issue), ,-, (2006).

[85] JH Oort \& HC van der Hulst. Gas and smoke in interstellar space. Bull Astron Inst Netherland, 10, 187-210, (1946).

[86] VS Safronov. Evolution of the protoplanetary cloud and formation of the earth and the planets. Israel program for scientific translations Ltd. Jerusalem, (1972).

[87] MN Popescu, JG Amar \& F Family. Rate-equation approach to island-size distributions and capture-numbers in submonolayer irreversible growth. Phys Rev B, 64, 205404, (2001).

[88] JA Blackman \& A Marshall. Coagulation and fragmentation in cluster-monomer reaction models. J Phys A; Math Gen, 27, 725-740, (1994). 